\title{
Solar Abundance Ratios of the Iron-Peak Elements in the Perseus Cluster
}

Hitomi Collaboration (2017) — Nature, 551, 478 (doi:10.1038/nature24301)

The metal abundance of the hot plasma that permeates galaxy clusters represents the accumulation of heavy elements produced by billions of supernovae ${ }^{1}$. Therefore, X-ray spectroscopy of the intracluster medium provides an opportunity to investigate the nature of supernova explosions integrated over cosmic time. In particular, the abundance of the iron-peak elements (chromium, manganese, iron and nickel) is key to understanding how the progenitors of typical type Ia supernovae evolve and explode ${ }^{\sqrt{2}+6}$. Recent X-ray studies of the intracluster medium found that the abundance ratios of these elements differ substantially from those seen in the $\mathrm{Sun}^{7 \cdot 11}$, suggesting differences between the nature of type Ia supernovae in the clusters and in the Milky Way. However, because the K-shell transition lines of chromium and manganese are weak and those of iron and nickel are very close in photon energy, high-resolution spectroscopy is required for an accurate determination of the abundances of these elements. Here we report observations of the Perseus cluster, with statistically significant detections of the resonance emission from chromium, manganese and nickel. Our measurements, combined with the latest atomic models, reveal that these elements have near-solar abundance ratios with respect to iron, in contrast to previous claims. Comparison between our results and modern nucleosynthesis calculations $12+14$ disfavours the hypothesis that type Ia supernova progenitors are exclusively white dwarfs with masses well below the Chandrasekhar limit (about 1.4 times the mass of the Sun). The observed abundance pattern of the iron-peak elements can be explained by taking into account a combination of near- and sub-Chandrasekhar-mass type Ia supernova systems, adding to the mounting evidence that both progenitor types make a substantial contribution to cosmic chemical enrichment $5,15,16$.

The Soft X-ray Spectrometer (SXS) on board Hitomi achieved unprecedented spectral resolution in orbit $(\Delta E \approx 5 \mathrm{eV} \text { in the } 2-10 \mathrm{keV} \text { band })^{17}$. Fig. 1 shows the SXS spectrum of the Perseus Cluster core $\left(r \lesssim 2^{\prime} \approx 40 \mathrm{kpc}\right)$ in the $1.8-9.0 \mathrm{keV}$ band. This was obtained from the same series of observations as our previous work that constrained turbulent velocities in the intracluster medium (ICM) $)^{17}$, but with $25 \%$ more exposure totaling $290 \mathrm{ks}$. The refined calibration of the telescope effective area and the SXS aperture window transmission now allows the first flux measurement of each individual line in the 1.8-9.0 keV band, encompassing the $\mathrm{H}$ - and He-like transitions from Si through Ni. 
The excellent performance of the SXS also makes possible the detection of weak resonance lines from $\mathrm{He}$-like $\mathrm{Cr}, \mathrm{Mn}$, and $\mathrm{Ni}$, with statistical significance of $6 \sigma, 4 \sigma$, and $12 \sigma$, respectively (Fig. 1b and 1c). Measurements of these line fluxes in celestial sources have been extremely challenging with traditional non-dispersive X-ray detectors (e.g., charge coupled devices, or CCDs), because such weak features readily blend into the bremsstrahlung continuum under lower spectral resolution and the Ni XXVII $\mathrm{He} \alpha$ and $\mathrm{Fe}$ XXV $\mathrm{He} \beta$ lines cannot be resolved (see Fig. 1c).

The hot ICM, confined in the deep cluster gravitational potential well, contains the dominant fraction $(\sim 80 \%)$ of metals in the cluster ${ }^{1}$. Among these, the Fe-peak elements $(\mathrm{Cr}, \mathrm{Mn}, \mathrm{Fe}$, and $\mathrm{Ni}$ ) are thought to be predominantly created by type Ia supernovae ( $\mathrm{SNe}$ Ia) occurring over a cosmological time period 18 . Therefore, the abundance of these elements provides crucial information about the integrated SN Ia nucleosynthesis and its relevant physics.

Despite the importance of SNe Ia as distance indicators in cosmology 20 , many of their fundamental aspects remain elusive. One important open question is whether the mass of an exploding white dwarf (WD) is close to the Chandrasekhar limit $\left(M_{\mathrm{Ch}} \approx 1.4 M_{\odot}\right)$, regardless of whether it originates from a single WD accreting mass from a non-degenerate companion ${ }^{22}$ or a violent merger of two WDs ${ }^{23}$. Recent hydrodynamical simulations show that both so-called delayed-detonation explosions of near- $M_{\mathrm{Ch}} \mathrm{WDs}^{4 / 12}$ and full detonations of sub- $M_{\mathrm{Ch}}$ WDs 13 [14 24 can reproduce the observed properties (such as optical light curves and spectra) of SNe Ia. Therefore, it is difficult to distinguish the two scenarios from optical observations of individual explosions alone.

From the point of view of SN Ia nucleosynthesis, the main difference between near- $M_{\mathrm{Ch}}$ and sub- $M_{\mathrm{Ch}}$ explosions is whether the WD core is dense enough for electron capture $\left(p+e^{-} \rightarrow n+\nu_{e}\right)$ to take place during the initial phase of the explosion. The threshold density for this reaction $\left(\rho_{c} \approx\right.$ $10^{8} \mathrm{~g} \mathrm{~cm}^{-3}$ ) is only achieved when the WD mass is close to $M_{\mathrm{Ch}}$. A distinguishing characteristic of the two models is, therefore, the production efficiency of neutron-rich species, like $\mathrm{Ni}$ and $\mathrm{Mn}$, that is higher in the near- $M_{\mathrm{Ch}}$ scenario ${ }^{4 / 6}$. We may exploit this distinction to identify the dominant type of SN Ia progenitors in galaxy clusters by measuring the abundance of the Fe-peak elements in the ICM. The results may apply globally, since rich galaxy clusters represent a scale sufficiently massive to be representative of the universe as a whole.

Here we model the SXS spectrum of the Perseus Cluster in the 1.8-9.0 keV band (Fig. 1a) with an optically thin thermal plasma in collisional ionization equilibrium using the latest atomic 
codes (AtomDB v.3.0.8 and SPEX v.3.03). The emission from the active galactic nucleus (AGN) of the cD galaxy NGC 1275 is taken into account by adding a power law and redshifted Fe $\mathrm{I} \mathrm{K} \alpha_{1}$ and $\mathrm{K} \alpha_{2}$ lines. Details about the analysis and systematic uncertainty assessment are described in the Methods section. Our constraints on the elemental abundances, with respect to Fe, are shown in Fig. 2 (red circles). These are fully consistent with the solar abundance ratios 25 .

Fig. 2 also plots previously measured ICM abundances of the Perseus Cluster core as well as the average of 44 objects including galaxy clusters, groups, and elliptical galaxies from XMM-Newton observations (blue triangles and squares) ${ }^{11}$. This plot highlights some important differences between the measurements. First, the SXS-measured abundances have statistical uncertainties comparable to the XMM-Newton results from the combined data of the 44 objects, despite a 15-times shorter exposure and a much smaller field of view. Second, while the abundances of $\mathrm{Si}, \mathrm{S}, \mathrm{Ar}$, and $\mathrm{Ca}$ are identical between the two studies, the earlier measurements systematically obtained supersolar abundances of the Fe-peak element from both the Perseus Cluster and the 44-object average.

Previous X-ray studies of clusters and elliptical galaxies often obtained a supersolar Ni/Fe ratio, leading the study authors to argue for differences in the nature of SNe Ia between the early-type galaxies and the Milky Way 3 . 10 . By contrast, optical spectra of old stars in early-type galaxies indicate that the relative abundances among the Fe-peak elements are consistent with the solar value (see yellow stars in Fig. 2) 2 . Our new X-ray measurement relieves this discrepancy and strongly suggests that the average nature of SNe Ia is independent of the star formation history of their host galaxies. This robust result, unaffected by complicated radiative transfer that may lend uncertainty to optical studies, is obtained by an accurate determination of the Ni abundance primarily based on the intensity of its resonance emission line that is easily resolved from the $\mathrm{Fe}$ $\mathrm{He} \beta$ line and other weak emission of Fe XXIV and Fe XXV.

Since $\mathrm{Cr}$ and $\mathrm{Mn}$ abundances of individual objects were not constrained by the previous XMM-Newton observations ${ }^{11}$, we cannot exclude the possibility that sample variance leads at least in part to the discrepancy between the two studies. Nevertheless, we demonstrate in Methods that high resolution spectroscopy is essential for robust measurements of these abundances. In short, only the SXS can clearly separate the weak resonance lines from the continuum component, enabling abundance measurements much less subject to systematic uncertainties in spectral modeling. The high-resolution SXS data have also stimulated the development of atomic models, reducing the uncertainties in the modeled line emissivities and improving the accuracy of the 
abundances with respect to the previous work.

Fig. 3 compares the SXS-measured abundances of the Fe-peak elements (black data points) with theoretical yields from the latest three-dimensional calculations of the near- $M_{\mathrm{Ch}} \mathrm{SN}$ Ia ${ }^{\sqrt{12}}$ (blue region) and sub- $M_{\mathrm{Ch}}$ merger ${ }^{13}$ (green region). We also consider a one-dimensional explosion of a single $1.0 M_{\odot} \mathrm{WD}^{14}$ (gray region) as an alternative example of a sub- $M_{\mathrm{Ch}} \mathrm{SN}$ Ia model. All of these models predict typical SN Ia brightness and a synthesized ${ }^{56} \mathrm{Ni}$ mass of $\sim 0.6 M_{\odot}$. In addition, contributions of core-collapse (CC) SNe are accounted for in each model given in the figure, utilizing mass-dependent yields ${ }^{27}$ averaged over the Salpeter initial mass function (IMF). We allow a conservatively wide range for the CC SN fraction, $f_{\mathrm{CC}} \equiv N_{\mathrm{CC}} /\left(N_{\mathrm{Ia}}+N_{\mathrm{CC}}\right)=0.6-0.9$ (typical for cluster cores $2.19 \sqrt[28]{29}$ ), instead of constraining an actual value from our observation (see Methods for more details). As expected, the near- $M_{\mathrm{Ch}}$ model predicts higher abundances of $\mathrm{Mn}$ and Ni owing to the efficient electron capture. The observed abundance pattern disfavors a hypothesis that all SNe Ia involve sub- $M_{\mathrm{Ch}} \mathrm{WD}$, and prefers the combination of the near- $M_{\mathrm{Ch}}$ and sub- $M_{\mathrm{Ch}} \mathrm{SNe}$ Ia with roughly equal numbers (red region in the figure). We also find that our result starkly contrasts with previous claims $\frac{37}{3}$, where introduction of rather non-standard full-deflagration $\mathrm{SN}$ Ia models was required to understand a $\mathrm{Ni} / \mathrm{Fe}$ ratio that was estimated to be much higher than our measurement. In Methods, we investigate other current SN Ia and CC models and find that our main conclusion remains valid, although an exact ratio of near- $M_{\mathrm{Ch}}$ to sub- $M_{\mathrm{Ch}}$ contributions may depend on the model details.

The Hitomi SXS observation has demonstrated the power of high-resolution X-ray spectroscopy: through measurement of the chemical enrichment of a single object, new insight has been gained into fundamental phenomena shaping the present-day universe. A common abundance pattern between the solar neighborhood and the Perseus Cluster suggests that the Sun's chemical composition is likely to be a good indicator of the average SN Ia nature in the universe. It is extremely important to scrutinize other environments like outskirts of galaxy clusters ${ }^{30}$ at high spectral resolution, a task left for future X-ray observatories. 


\section{References}

1. Mushotzky, R. et al. Measurement of the Elemental Abundances in Four Rich Clusters of Galaxies. I. Observations. Astrophys. J. 466, 686 (1996).

2. Finoguenov, A., Matsushita, K., Böhringer, H., Ikebe, Y. \& Arnaud, M. X-ray evidence for spectroscopic diversity of type Ia supernovae:. XMM observation of the elemental abundance pattern in M 87. Astron. Astrophys. 381, 21-31 (2002).

3. Mernier, F. et al. Origin of central abundances in the hot intra-cluster medium. II. Chemical enrichment and supernova yield models. Astron. Astrophys. 595, A126 (2016).

4. Maeda, K. et al. Nebular Spectra and Explosion Asymmetry of Type Ia Supernovae. Astrophys. J. 708, 1703-1715 (2010).

5. Seitenzahl, I. R., Cescutti, G., Röpke, F. K., Ruiter, A. J. \& Pakmor, R. Solar abundance of manganese: a case for near Chandrasekhar-mass Type Ia supernova progenitors. Astron. Astrophys. 559, L5 (2013).

6. Yamaguchi, H. et al. A Chandrasekhar Mass Progenitor for the Type Ia Supernova Remnant 3C 397 from the Enhanced Abundances of Nickel and Manganese. Astrophys. J. Lett. 801, L31 (2015).

7. Dupke, R. A. \& White, R. E., III. Constraints on Type IA Supernova Models from X-Ray Spectra of Galaxy Clusters. Astrophys. J. 528, 139-144 (2000).

8. Gastaldello, F. \& Molendi, S. Ni Abundance in the Core of the Perseus Cluster: An Answer to the Significance of Resonant Scattering. Astrophys. J. 600, 670-680 (2004).

9. de Plaa, J. et al. Constraining supernova models using the hot gas in clusters of galaxies. Astron. Astrophys. 465, 345-355 (2007).

10. de Grandi, S. \& Molendi, S. Metal abundances in the cool cores of galaxy clusters. Astron. Astrophys. 508, 565-574 (2009).

11. Mernier, F. et al. Origin of central abundances in the hot intra-cluster medium. I. Individual and average abundance ratios from XMM-Newton EPIC. Astron. Astrophys. 592, A157 (2016).

12. Seitenzahl, I. R. et al. Three-dimensional delayed-detonation models with nucleosynthesis for Type Ia supernovae. Mon. Not. R. Astron. Soc. 429, 1156-1172 (2013). 
13. Pakmor, R. et al. Normal Type Ia Supernovae from Violent Mergers of White Dwarf Binaries. Astrophys. J. Lett. 747, L10 (2012).

14. Woosley, S. E. \& Kasen, D. Sub-Chandrasekhar Mass Models for Supernovae. Astrophys. J. 734, 38 (2011).

15. Scalzo, R. A., Ruiter, A. J. \& Sim, S. A. The ejected mass distribution of Type Ia supernovae: a significant rate of non-Chandrasekhar-mass progenitors. Mon. Not. R. Astron. Soc. 445, 2535-2544 (2014).

16. Blondin, S., Dessart, L., Hillier, D. J. \& Khokhlov, A. M. Evidence for sub-Chandrasekhar-mass progenitors of Type Ia supernovae at the faint end of the width-luminosity relation. Mon. Not. R. Astron. Soc. 470, 157-165 (2017).

17. Hitomi Collaboration. The quiescent intracluster medium in the core of the Perseus cluster. Nature 535, 117-121 (2016).

18. Loewenstein, M. \& Mushotzky, R. F. Measurement of the Elemental Abundances in Four Rich Clusters of Galaxies. II. The Initial Mass Function and Mass Loss in Elliptical Galaxies, Enrichment, and Energetics in the ICM. Astrophys. J. 466, 695 (1996).

19. Matsushita, K., Sakuma, E., Sasaki, T., Sato, K. \& Simionescu, A. Metal-mass-to-light Ratios of the Perseus Cluster Out to the Virial Radius. Astrophys. J. 764, 147 (2013).

20. Riess, A. G. et al. Observational Evidence from Supernovae for an Accelerating Universe and a Cosmological Constant. Astron. J. 116, 1009-1038 (1998).

21. Perlmutter, S. et al. Measurements of $\Omega$ and $\Lambda$ from 42 High-Redshift Supernovae. Astrophys. J. 517, 565-586 (1999).

22. Whelan, J. \& Iben, I., Jr. Binaries and Supernovae of Type I. Astrophys. J. 186, 1007-1014 (1973).

23. Webbink, R. F. Double white dwarfs as progenitors of R Coronae Borealis stars and Type I supernovae. Astrophys. J. 277, 355-360 (1984).

24. Sim, S. A. et al. Detonations in Sub-Chandrasekhar-mass C+O White Dwarfs. Astrophys. J. Lett. 714, L52-L57 (2010).

25. Lodders, K., Palme, H. \& Gail, H.-P. Abundances of the Elements in the Solar System. Landolt Börnstein (2009). 
26. Conroy, C., Graves, G. J. \& van Dokkum, P. G. Early-type Galaxy Archeology: Ages, Abundance Ratios, and Effective Temperatures from Full-spectrum Fitting. Astrophys. J. 780, 33 (2014).

27. Nomoto, K., Kobayashi, C. \& Tominaga, N. Nucleosynthesis in Stars and the Chemical Enrichment of Galaxies. Annual Rev. Astron. Astrophys. 51, 457-509 (2013).

28. Sato, K. et al. Type Ia and II Supernovae Contributions to Metal Enrichment in the Intracluster Medium Observed with Suzaku. Astrophys. J. Lett. 667, L41-L44 (2007).

29. Bulbul, E., Smith, R. K. \& Loewenstein, M. A New Method to Constrain Supernova Fractions Using X-Ray Observations of Clusters of Galaxies. Astrophys. J. 753, 54 (2012).

30. Werner, N., Urban, O., Simionescu, A. \& Allen, S. W. A uniform metal distribution in the intergalactic medium of the Perseus cluster of galaxies. Nature 502, 656-658 (2013). 


\section{References for Methods}

31. Eckart, M. E. et al. Ground calibration of the Astro-H (Hitomi) soft x-ray spectrometer. In Society of Photo-Optical Instrumentation Engineers (SPIE) Conference Series, vol. 9905 of Proc. SPIE, 99053W (2016).

32. Angelini, L. et al. Astro-H data analysis, processing and archive. In Space Telescopes and Instrumentation 2016: Ultraviolet to Gamma Ray, vol. 9905 of Proc. SPIE, 990514 (2016).

33. Leutenegger, M. A. et al. In-flight verification of the calibration and performance of the ASTRO-H (Hitomi) Soft X-Ray Spectrometer. In Society of Photo-Optical Instrumentation Engineers (SPIE) Conference Series, vol. 9905 of Proc. SPIE, 99053 U (2016).

34. Kelley, R. L. et al. The Astro-H high resolution soft x-ray spectrometer. In Society of Photo-Optical Instrumentation Engineers (SPIE) Conference Series, vol. 9905 of Proc. SPIE, 99050V (2016).

35. Porter, F. S. et al. In-flight performance of the Soft X-ray Spectrometer detector system on Astro-H. In Society of Photo-Optical Instrumentation Engineers (SPIE) Conference Series, vol. 9905 of Proc. SPIE, 99050W (2016).

36. Ferruit, P., Adam, G., Binette, L. \& Pécontal, E. TIGER observations of the low and high velocity components of NGC 1275. NEWA 2, 345-363 (1997).

37. Okajima, T. et al. First peek of ASTRO-H Soft X-ray Telescope (SXT) in-orbit performance. In Society of Photo-Optical Instrumentation Engineers (SPIE) Conference Series, vol. 9905 of Proc. SPIE, $99050 Z$ (2016).

38. Cash, W. Parameter estimation in astronomy through application of the likelihood ratio. Astrophys. J. 228, 939-947 (1979).

39. Tamura, T. et al. X-ray Spectroscopy of the Core of the Perseus Cluster with Suzaku: Elemental Abundances in the Intracluster Medium. Astrophys. J. Lett. 705, L62-L66 (2009).

40. Foster, A. R., Ji, L., Smith, R. K. \& Brickhouse, N. S. Updated Atomic Data and Calculations for X-Ray Spectroscopy. Astrophys. J. 756, 128 (2012).

41. Churazov, E., Forman, W., Jones, C., Sunyaev, R. \& Böhringer, H. XMM-Newton observations of the Perseus cluster - II. Evidence for gas motions in the core. Mon. Not. R. Astron. Soc. 347, 29-35 (2004). 
42. Kalberla, P. M. W. et al. The Leiden/Argentine/Bonn (LAB) Survey of Galactic HI. Final data release of the combined LDS and IAR surveys with improved stray-radiation corrections. Astron. Astrophys. 440, 775-782 (2005).

43. Zhuravleva, I. et al. Resonant scattering in the Perseus Cluster: spectral model for constraining gas motions with Astro-H. Mon. Not. R. Astron. Soc. 435, 3111-3121 (2013).

44. Sanders, J. S. \& Fabian, A. C. A deeper X-ray study of the core of the Perseus galaxy cluster: the power of sound waves and the distribution of metals and cosmic rays. Mon. Not. R. Astron. Soc. 381, 1381-1399 (2007).

45. Kaastra, J. S., Mewe, R. \& Nieuwenhuijzen, H. SPEX: a new code for spectral analysis of X \& UV spectra. In Yamashita, K. \& Watanabe, T. (eds.) UV and X-ray Spectroscopy of Astrophysical and Laboratory Plasmas, 411-414 (1996).

46. Simionescu, A. et al. Chemical enrichment in the cluster of galaxies Hydra A. Astron. Astrophys. 493, 409-424 (2009).

47. Ezer, C. et al. Uniform Contribution of Supernova Explosions to the Chemical Enrichment of Abell 3112 out to $\mathrm{R}_{200}$. Astrophys. J. 836, 110 (2017).

48. Mulchaey, J. S., Kasliwal, M. M. \& Kollmeier, J. A. Calcium-rich Gap Transients: Solving the Calcium Conundrum in the Intracluster Medium. Astrophys. J. Lett. 780, L34 (2014).

49. Fink, M. et al. Three-dimensional pure deflagration models with nucleosynthesis and synthetic observables for Type Ia supernovae. Mon. Not. R. Astron. Soc. 438, 1762-1783 (2014).

50. Travaglio, C., Hillebrandt, W., Reinecke, M. \& Thielemann, F.-K. Nucleosynthesis in multi-dimensional SN Ia explosions. Astron. Astrophys. 425, 1029-1040 (2004).

51. Travaglio, C., Röpke, F. K., Gallino, R. \& Hillebrandt, W. Type Ia Supernovae as Sites of the p-process: Two-dimensional Models Coupled to Nucleosynthesis. Astrophys. J. 739, 93 (2011).

52. Bravo, E. \& Martínez-Pinedo, G. Sensitivity study of explosive nucleosynthesis in type Ia supernovae: Modification of individual thermonuclear reaction rates. Phys. Rev. C 85, 055805 (2012).

53. Tsujimoto, T. \& Shigeyama, T. Diversity of Type Ia Supernovae Imprinted in Chemical Abundances. Astrophys. J. Lett. 760, L38 (2012). 
54. Langanke, K. \& Martínez-Pinedo, G. Shell-model calculations of stellar weak interaction rates: II. Weak rates for nuclei in the mass range $/ A=45-65$ in supernovae environments. Nuclear Physics A 673, 481-508 (2000). 
Acknowledgements Acknowledgements are provided in the Supplementary Information.

Author Contributions H. Yamaguchi wrote the manuscript. H. Yamaguchi, S. Nakashima, A. Simionescu, E. Bulbul, and M. Loewenstein analyzed the data specifically for this project. H. Yamaguchi, K. Matsushita, M. Loewensterin, A. Simionescu, S. Nakashima, K. Sato, and R. Mushotzky discussed the results. Y. Ishisaki confirmed the reliability of the observed results based on his expertise in the SXS signal processing system. The science goals of Hitomi were discussed and developed over more than 10 years by the ASTRO-H Science Working Group (SWG), all members of which are authors of this manuscript. All the instruments were prepared by joint efforts of the team. Calibration of the Perseus dataset was carried out by members of the SXS team. The manuscript was subject to an internal collaboration-wide review process. All authors reviewed and approved the final version of the manuscript.

Author Information Reprints and permissions information is available at www.nature.com/reprints. The authors declare no competing financial interests. Correspondence and requests for materials should be addressed to H. Yamaguchi (hiroya.yamaguchi@nasa.gov) and K. Matsushita (matusita@rs.kagu.tus.ac.jp).

Hitomi Collaboration Felix Aharonian ${ }^{1,2,3}$, Hiroki Akamatsu ${ }^{4}$, Fumie Akimoto ${ }^{5}$, Steven W. Allen ${ }^{6,7,8}$, Lorella Angelini $^{9}$, Marc Audard ${ }^{10}$, Hisamitsu Awaki ${ }^{11}$, Magnus Axelsson ${ }^{12}$, Aya Bamba ${ }^{13,14}$, Marshall W. Bautz ${ }^{15}$, Roger Blandford, ${ }^{6,7,8}$, Laura W. Brenneman ${ }^{16}$, Gregory V. Brown ${ }^{17}$, Esra Bulbul ${ }^{15,16}$, Edward M. Cackett ${ }^{18}$, Maria Chernyakova ${ }^{1}$, Meng P. Chiao ${ }^{9}$, Paolo S. Coppi ${ }^{19,20}$, Elisa Costantini ${ }^{4}$, Jelle de Plaa ${ }^{4}$, Jan-Willem den Herder ${ }^{4}$, Chris Done ${ }^{21}$, Tadayasu Dotani ${ }^{22}$, Ken Ebisawa ${ }^{22}$, Megan E. Eckart $^{9}$, Teruaki Enoto ${ }^{23,24}$, Yuichiro Ezoe ${ }^{25}$, Andrew C. Fabian ${ }^{26}$, Carlo Ferrigno ${ }^{10}$, Adam R. Foster ${ }^{16}$, Ryuichi Fujimoto $^{27}$, Yasushi Fukazawa ${ }^{28}$, Akihiro Furuzawa ${ }^{29}$, Massimiliano Galeazzi ${ }^{30}$, Luigi C. Gallo ${ }^{31}$, Poshak Gandhi ${ }^{32}$, Margherita Giustini ${ }^{4}$, Andrea Goldwurm ${ }^{33,34}$, Liyi Gu ${ }^{4}$, Matteo Guainazzi ${ }^{35}$, Yoshito Haba $^{36}$, Kouichi Hagino ${ }^{37}$, Kenji Hamaguchi ${ }^{9,38}$, Ilana M. Harrus ${ }^{9,38}$, Isamu Hatsukade ${ }^{39}$, Katsuhiro Hayashi $^{22,40}$, Takayuki Hayashi ${ }^{40}$, Kiyoshi Hayashida ${ }^{41}$, Junko S. Hiraga ${ }^{42}$, Ann Hornschemeier ${ }^{9}$, Akio Hoshino $^{43}$, John P. Hughes ${ }^{44}$, Yuto Ichinohe ${ }^{25}$, Ryo Iizuka ${ }^{22}$, Hajime Inoue ${ }^{45}$, Yoshiyuki Inoue ${ }^{22}$, Manabu Ishida ${ }^{22}$, Kumi Ishikawa ${ }^{22}$, Yoshitaka Ishisaki ${ }^{25}$, Masachika Iwai ${ }^{22}$, Jelle Kaastra ${ }^{4,46}$, Tim Kallman $^{9}$, Tsuneyoshi Kamae ${ }^{13}$, Jun Kataoka ${ }^{47}$, Satoru Katsuda ${ }^{48}$, Nobuyuki Kawai ${ }^{49}$, Richard L. Kelley ${ }^{9}$, Caroline A. Kilbourne ${ }^{9}$, Takao Kitaguchi ${ }^{28}$, Shunji Kitamoto ${ }^{43}$, Tetsu Kitayama ${ }^{50}$, Takayoshi Kohmura ${ }^{37}$, Motohide Kokubun $^{22}$, Katsuji Koyama ${ }^{51}$, Shu Koyama ${ }^{22}$, Peter Kretschmar ${ }^{52}$, Hans A. Krimm ${ }^{53,54}$, Aya Kubota $^{55}$, Hideyo Kunieda ${ }^{40}$, Philippe Laurent ${ }^{33,34}$, Shiu-Hang Lee ${ }^{23}$, Maurice A. Leutenegger ${ }^{9,38}$, Olivier Limousine $^{34}$, Michael Loewenstein ${ }^{9,56}$, Knox S. Long ${ }^{57}$, David Lumb ${ }^{35}$, Greg Madejski ${ }^{6}$, Yoshitomo Maeda $^{22}$, Daniel Maier ${ }^{33,34}$, Kazuo Makishima ${ }^{58}$, Maxim Markevitch ${ }^{9}$, Hironori Matsumoto ${ }^{41}$, Kyoko Matsushita $^{59}$, Dan McCammon $^{60}$, Brian R. McNamara ${ }^{61}$, Missagh Mehdipour ${ }^{4}$, Eric D. Miller ${ }^{15}$, Jon M. Miller ${ }^{62}$, Shin Mineshige ${ }^{23}$, Kazuhisa Mitsuda ${ }^{22}$, Ikuyuki Mitsuishi ${ }^{40}$, Takuya Miyazawa ${ }^{63}$, Tsunefumi Mizuno ${ }^{28,64}$, Hideyuki Mori ${ }^{9}$, Koji Mori ${ }^{39}$, Koji Mukai ${ }^{9}, 38$, Hiroshi Murakami ${ }^{65}$, Richard F. Mushotzky ${ }^{56}$, Takao Nakagawa ${ }^{22}$, Hiroshi Nakajima ${ }^{41}$, Takeshi Nakamori ${ }^{66}$, Shinya Nakashima ${ }^{58}$, 
Kazuhiro Nakazawa ${ }^{13,14}$, Kumiko K. Nobukawa ${ }^{67}$, Masayoshi Nobukawa ${ }^{68}$, Hirofumi Noda ${ }^{69,70}$, Hirokazu Odaka $^{6}$, Takaya Ohashi ${ }^{25}$, Masanori Ohno ${ }^{28}$, Takashi Okajima ${ }^{9}$, Naomi Ota ${ }^{67}$, Masanobu Ozaki ${ }^{22}$, Frits Paerels $^{71}$, Stéphane Paltani ${ }^{10}$, Robert Petre ${ }^{9}$, Ciro Pinto ${ }^{26}$, Frederick S. Porter ${ }^{9}$, Katja Pottschmidt ${ }^{9,38}$, Christopher S. Reynolds ${ }^{56}$, Samar Safi-Harb ${ }^{72}$, Shinya Saito ${ }^{43}$, Kazuhiro Sakai ${ }^{9}$, Toru Sasaki ${ }^{59}$, Goro Sato $^{22}$, Kosuke Sato ${ }^{59}$, Rie Sato ${ }^{22}$, Makoto Sawada ${ }^{73}$, Norbert Schartel ${ }^{52}$, Peter J. Serlemitsos ${ }^{9}$, Hiromi Seta $^{25}$, Megumi Shidatsu ${ }^{58}$, Aurora Simionescu ${ }^{22}$, Randall K. Smith ${ }^{16}$, Yang Soong ${ }^{9}$, Łukasz Stawarz ${ }^{74}$, Yasuharu Sugawara $^{22}$, Satoshi Sugita ${ }^{49}$, Andrew Szymkowiak ${ }^{20}$, Hiroyasu Tajima ${ }^{5}$, Hiromitsu Takahashi ${ }^{28}$, Tadayuki Takahashi ${ }^{22}$, Shin'ichiro Takeda ${ }^{63}$, Yoh Takei ${ }^{22}$, Toru Tamagawa ${ }^{75}$, Takayuki Tamura ${ }^{22}$, Takaaki Tanaka $^{51}$, Yasuo Tanaka ${ }^{76,22}$, Yasuyuki T. Tanaka ${ }^{28}$, Makoto S. Tashiro ${ }^{77}$, Yuzuru Tawara ${ }^{40}$, Yukikatsu Terada $^{77}$, Yuichi Terashima ${ }^{11}$, Francesco Tombesi ${ }^{9,56,78}$, Hiroshi Tomida ${ }^{22}$, Yohko Tsuboi ${ }^{48}$, Masahiro Tsujimoto $^{22}$, Hiroshi Tsunemi ${ }^{41}$, Takeshi Go Tsuru ${ }^{51}$, Hiroyuki Uchida ${ }^{51}$, Hideki Uchiyama ${ }^{79}$, Yasunobu Uchiyama $^{43}$, Shutaro Ueda ${ }^{22}$, Yoshihiro Ueda ${ }^{23}$, Shin'ichiro Uno ${ }^{80}$, C. Megan Urry ${ }^{20}$, Eugenio Ursino ${ }^{30}$, Cor P. de Vries ${ }^{4}$, Shin Watanabe ${ }^{22}$, Norbert Werner ${ }^{81,82,28}$, Daniel R. Wik ${ }^{83,9,84}$, Dan R. Wilkins ${ }^{6}$, Brian J. Williams ${ }^{57}$, Shinya Yamada ${ }^{25}$, Hiroya Yamaguchi ${ }^{9,56}$, Kazutaka Yamaoka ${ }^{5,40}$, Noriko Y. Yamasaki ${ }^{22}$, Makoto Yamauchi ${ }^{39}$, Shigeo Yamauchi ${ }^{67}$, Tahir Yaqoob ${ }^{9,38}$, Yoichi Yatsu ${ }^{49}$, Daisuke Yonetoku ${ }^{27}$, Irina Zhuravleva $^{6,7}$, Abderahmen Zoghbi ${ }^{62}$

${ }^{1}$ Dublin Institute for Advanced Studies, 31 Fitzwilliam Place, Dublin 2, Ireland

${ }^{2}$ Max-Planck-Institut für Kernphysik, P.O. Box 103980, 69029 Heidelberg, Germany

${ }^{3}$ Gran Sasso Science Institute, viale Francesco Crispi, 767100 L'quila (AQ), Italy

${ }^{4}$ SRON Netherlands Institute for Space Research, Sorbonnelaan 2, 3584 CA Utrecht, The Netherlands

${ }^{5}$ Institute for Space-Earth Environmental Research, Nagoya University, Furo-cho, Chikusa-ku, Nagoya, Aichi 464-8601, Japan

${ }^{6}$ Kavli Institute for Particle Astrophysics and Cosmology, Stanford University, 452 Lomita Mall, Stanford, CA 94305, USA

${ }^{7}$ Department of Physics, Stanford University, 382 Via Pueblo Mall, Stanford, CA 94305, USA

${ }^{8}$ SLAC National Accelerator Laboratory, 2575 Sand Hill Road, Menlo Park, CA 94025, USA

${ }^{9}$ NASA, Goddard Space Flight Center, 8800 Greenbelt Road, Greenbelt, MD 20771, USA

${ }^{10}$ Department of Astronomy, University of Geneva, ch. d'Écogia 16, CH-1290 Versoix, Switzerland

${ }^{11}$ Department of Physics, Ehime University, Bunkyo-cho, Matsuyama, Ehime 790-8577, Japan

${ }^{12}$ Department of Physics and Oskar Klein Center, Stockholm University, 10691 Stockholm, Sweden

${ }^{13}$ Department of Physics, The University of Tokyo, 7-3-1 Hongo, Bunkyo-ku, Tokyo 113-0033, Japan 
${ }^{14}$ Research Center for the Early Universe, School of Science, The University of Tokyo, 7-3-1 Hongo, Bunkyo-ku, Tokyo 113-0033, Japan

${ }^{15}$ Kavli Institute for Astrophysics and Space Research, Massachusetts Institute of Technology, 77 Massachusetts Avenue, Cambridge, MA 02139, USA

${ }^{16}$ Smithsonian Astrophysical Observatory, 60 Garden St., MS-4. Cambridge, MA 02138, USA

${ }^{17}$ Lawrence Livermore National Laboratory, 7000 East Avenue, Livermore, CA 94550, USA

${ }^{18}$ Department of Physics and Astronomy, Wayne State University, 666 W. Hancock St, Detroit, MI 48201, USA

${ }^{19}$ Astronomy Department, Yale University, New Haven, CT 06520-8101, USA

${ }^{20}$ Physics Department, Yale University, New Haven, CT 06520-8120, USA

${ }^{21}$ Centre for Extragalactic Astronomy, Department of Physics, University of Durham, South Road, Durham, DH1 3LE, UK

${ }^{22}$ Japan Aerospace Exploration Agency, Institute of Space and Astronautical Science, 3-1-1 Yoshino-dai, Chuo-ku, Sagamihara, Kanagawa 252-5210, Japan

${ }^{23}$ Department of Astronomy, Kyoto University, Kitashirakawa-Oiwake-cho, Sakyo-ku, Kyoto 606-8502, Japan

${ }^{24}$ The Hakubi Center for Advanced Research, Kyoto University, Kyoto 606-8302, Japan

${ }^{25}$ Department of Physics, Tokyo Metropolitan University, 1-1 Minami-Osawa, Hachioji, Tokyo 192-0397, Japan

${ }^{26}$ Institute of Astronomy, University of Cambridge, Madingley Road, Cambridge, CB3 OHA, UK

${ }^{27}$ Faculty of Mathematics and Physics, Kanazawa University, Kakuma-machi, Kanazawa, Ishikawa 920-1192, Japan

${ }^{28}$ School of Science, Hiroshima University, 1-3-1 Kagamiyama, Higashi-Hiroshima 739-8526, Japan

${ }^{29}$ Fujita Health University, Toyoake, Aichi 470-1192, Japan

${ }^{30}$ Physics Department, University of Miami, 1320 Campo Sano Dr., Coral Gables, FL 33146, USA

${ }^{31}$ Department of Astronomy and Physics, Saint Mary’s University, 923 Robie Street, Halifax, NS, B3H 3C3, Canada

${ }^{32}$ Department of Physics and Astronomy, University of Southampton, Highfield, Southampton, SO17 1BJ, UK

${ }^{33}$ Laboratoire APC, 10 rue Alice Domon et Léonie Duquet, 75013 Paris, France

${ }^{34}$ CEA Saclay, 91191 Gif sur Yvette, France

${ }^{35}$ European Space Research and Technology Center, Keplerlaan 12201 AZ Noordwijk, The Netherlands

${ }^{36}$ Department of Physics and Astronomy, Aichi University of Education, Aichi 448-8543, Japan 
${ }^{37}$ Department of Physics, Tokyo University of Science, 2641 Yamazaki, Noda, Chiba, 278-8510, Japan

${ }^{38}$ Department of Physics, University of Maryland Baltimore County, 1000 Hilltop Circle, Baltimore, MD 21250, USA

${ }^{39}$ Department of Applied Physics and Electronic Engineering, University of Miyazaki, 1-1 Gakuen Kibanadai-Nishi, Miyazaki, 889-2192, Japan

${ }^{40}$ Department of Physics, Nagoya University, Furo-cho, Chikusa-ku, Nagoya, Aichi 464-8602, Japan

${ }^{41}$ Department of Earth and Space Science, Osaka University, 1-1 Machikaneyama-cho, Toyonaka, Osaka 560-0043, Japan

${ }^{42}$ Department of Physics, Kwansei Gakuin University, 2-1 Gakuen, Sanda, Hyogo 669-1337, Japan

${ }^{43}$ Department of Physics, Rikkyo University, 3-34-1 Nishi-Ikebukuro, Toshima-ku, Tokyo 171-8501, Japan

${ }^{44}$ Department of Physics and Astronomy, Rutgers University, 136 Frelinghuysen Road, Piscataway, NJ 08854, USA

${ }^{45}$ Meisei University, 2-1-1 Hodokubo, Hino, Tokyo 191-8506, Japan

${ }^{46}$ Leiden Observatory, Leiden University, PO Box 9513, 2300 RA Leiden, the Netherlands

${ }^{47}$ Research Institute for Science and Engineering, Waseda University, 3-4-1 Ohkubo, Shinjuku, Tokyo, 169-8555, Japan

${ }^{48}$ Department of Physics, Chuo University, 1-13-27 Kasuga, Bunkyo, Tokyo 112-8551, Japan

${ }^{49}$ Department of Physics, Tokyo Institute of Technology, 2-12-1 Ookayama, Meguro-ku, Tokyo 152-8550, Japan

${ }^{50}$ Department of Physics, Toho University, 2-2-1 Miyama, Funabashi, Chiba 274-8510, Japan

${ }^{51}$ Department of Physics, Kyoto University, Kitashirakawa-Oiwake-Cho, Sakyo, Kyoto 606-8502, Japan

${ }^{52}$ European Space Astronomy Center, Camino Bajo del Castillo, s/n., 28692 Villanueva de la Cañada, Madrid, Spain

${ }^{53}$ Universities Space Research Association, 7178 Columbia Gateway Drive, Columbia, MD 21046, USA

${ }^{54}$ National Science Foundation, 4201 Wilson Blvd, Arlington, VA 22230, USA

${ }^{55}$ Department of Electronic Information Systems, Shibaura Institute of Technology, 307 Fukasaku, Minuma-ku, Saitama-shi, Saitama 337-8570, Japan

${ }^{56}$ Department of Astronomy, University of Maryland, College Park, MD 20742, USA

${ }^{57}$ Space Telescope Science Institute, 3700 San Martin Drive, Baltimore, MD 21218, USA

${ }^{58}$ Institute of Physical and Chemical Research, 2-1 Hirosawa, Wako, Saitama 351-0198 
${ }^{59}$ Department of Physics, Tokyo University of Science, 1-3 Kagurazaka, Shinjuku-ku, Tokyo 162-8601, Japan

${ }^{60}$ Department of Physics, University of Wisconsin, Madison, WI 53706, USA

${ }^{61}$ Department of Physics and Astronomy, University of Waterloo, 200 University Avenue West, Waterloo, Ontario, N2L 3G1, Canada

${ }^{62}$ Department of Astronomy, University of Michigan, 1085 South University Avenue, Ann Arbor, MI 48109, USA

${ }^{63}$ Okinawa Institute of Science and Technology Graduate University, 1919-1 Tancha, Onna-son Okinawa, 904-0495, Japan

${ }^{64}$ Hiroshima Astrophysical Science Center, Hiroshima University, Higashi-Hiroshima, Hiroshima 739-8526, Japan

${ }^{65}$ Faculty of Liberal Arts, Tohoku Gakuin University, 2-1-1 Tenjinzawa, Izumi-ku, Sendai, Miyagi 981-3193

${ }^{66}$ Faculty of Science, Yamagata University, 1-4-12 Kojirakawa-machi, Yamagata, Yamagata 990-8560, Japan

${ }^{67}$ Department of Physics, Nara Women's University, Kitauoyanishi-machi, Nara, Nara 630-8506, Japan

${ }^{68}$ Department of Teacher Training and School Education, Nara University of Education, Takabatake-cho, Nara, Nara 630-8528, Japan

${ }^{69}$ Frontier Research Institute for Interdisciplinary Sciences, Tohoku University, 6-3 Aramakiazaaoba, Aoba-ku, Sendai, Miyagi 980-8578, Japan

${ }^{70}$ Astronomical Institute, Tohoku University, 6-3 Aramakiazaaoba, Aoba-ku, Sendai, Miyagi 980-8578, Japan

${ }^{71}$ Astrophysics Laboratory, Columbia University, 550 West 120th Street, New York, NY 10027, USA

${ }^{72}$ Department of Physics and Astronomy, University of Manitoba, Winnipeg, MB R3T 2N2, Canada

${ }^{73}$ Department of Physics and Mathematics, Aoyama Gakuin University, 5-10-1 Fuchinobe, Chuo-ku, Sagamihara, Kanagawa 252-5258, Japan

${ }^{74}$ Astronomical Observatory of Jagiellonian University, ul. Orla 171, 30-244 Kraków, Poland

${ }^{75}$ RIKEN Nishina Center, 2-1 Hirosawa, Wako, Saitama 351-0198, Japan

${ }^{76}$ Max Planck Institute for extraterrestrial Physics, Giessenbachstrasse 1, 85748 Garching, Germany

${ }^{77}$ Department of Physics, Saitama University, 255 Shimo-Okubo, Sakura-ku, Saitama, 338-8570, Japan 
${ }^{78}$ Department of Physics, University of Rome "Tor Vergata", Via della Ricerca Scientifica 1, I-00133 Rome, Italy

${ }^{79}$ Faculty of Education, Shizuoka University, 836 Ohya, Suruga-ku, Shizuok a 422-8529, Japan

${ }^{80}$ Faculty of Health Sciences, Nihon Fukushi University, 26-2 Higashi Haemi-cho, Handa, Aichi 475-0012, Japan

${ }^{81}$ MTA-Eötvös University Lendület Hot Universe Research Group, Pázmány Péter sétány 1/A, Budapest, 1117, Hungary

${ }^{82}$ Department of Theoretical Physics and Astrophysics, Faculty of Science, Masaryk University, Kotlářská 2, Brno, 611 37, Czech Republic

${ }^{83}$ Department of Physics and Astronomy, University of Utah, 115 South 1400 East, Salt Lake City, Utah 84112, USA

${ }^{84}$ The Johns Hopkins University, Homewood Campus, Baltimore, MD 21218, USA 

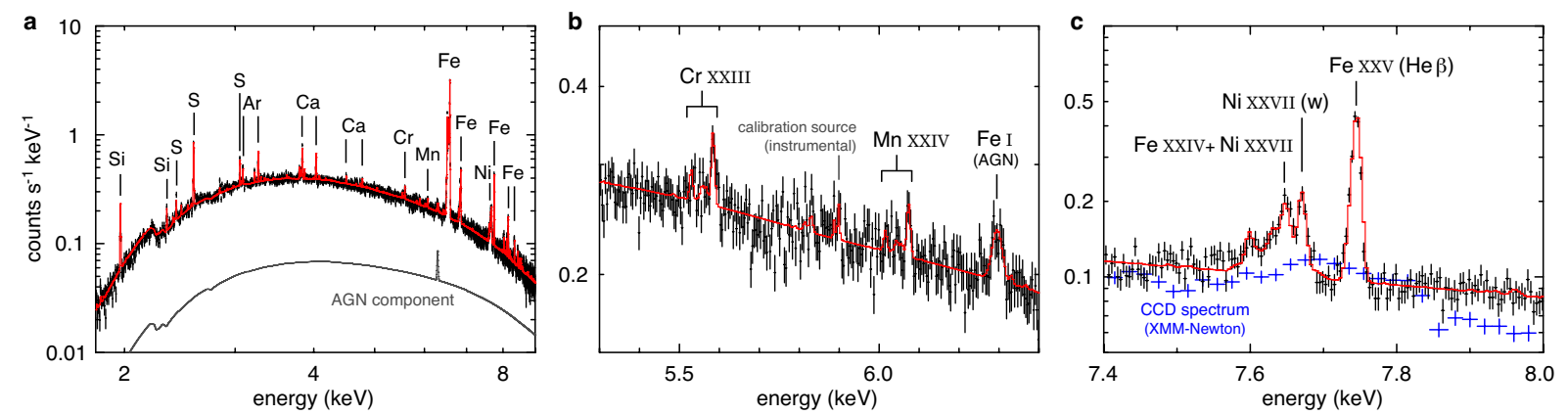

Figure 1: The Hitomi/SXS spectra of the Perseus Cluster. (a) The spectrum (black) in the $1.8-9.0 \mathrm{keV}$ band modeled with an optically thin thermal plasma based on the atomic code AtomDB (red). The error bars are at a $1 \sigma$ confidence level. The emission from NGC 1275 (AGN) is indicated by the gray curve. The spectrum is rebinned by $4 \mathrm{eV}$ for clarity, though $1-\mathrm{eV}$ bins were used for fitting. (b) The zoom-in spectrum in the 5.3-6.4 keV band, where the emission from He-like $\mathrm{Cr}$ and $\mathrm{Mn}$ are detected. The red-shifted Fe I fluorescence from the AGN is resolved as well. (c) The same in the 7.4-8.0 keV band, highlighting the Ni XXVII resonance (w) line clearly separated from the stronger Fe XXV $\mathrm{He} \beta$ and other emission. This enables the first accurate measurement of the Ni abundance in a galaxy cluster. For comparison, an XMM-Newton spectrum extracted from the same spatial region is shown as the blue data points. 


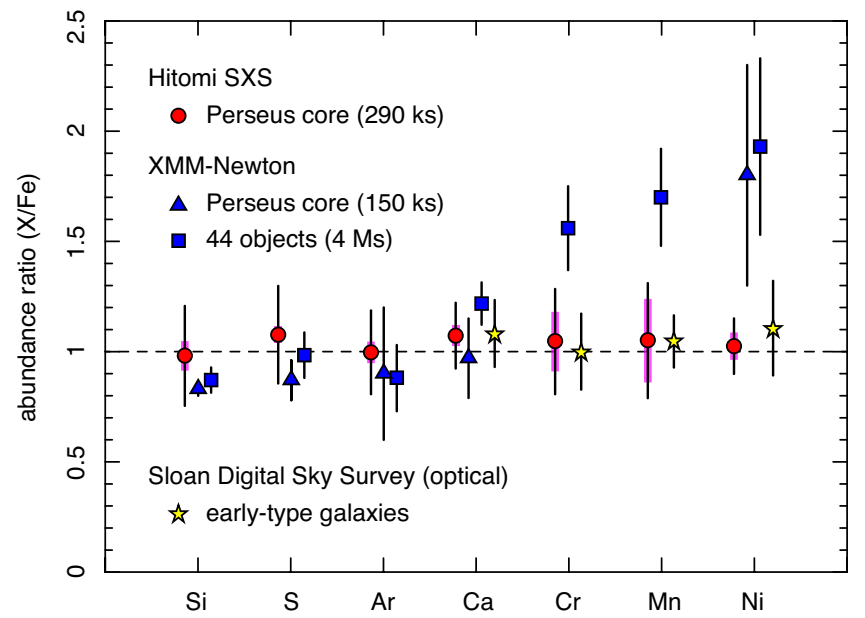

Figure 2: Elemental abundances of the Perseus Cluster. The values are relative to the solar abundances ${ }^{25}$ with respect to Fe. The red circles represent the SXS measurements with error bars of typical statistical uncertainty at a $1 \sigma$ confidence level (thick magenta) and systematic uncertainty due to the model selection (thin black: see Methods for details). The blue triangles and squares represent the XMM-Newton results from the Perseus Cluster core and the integrated data of 44 objects, respectively 11 . The yellow stars show the optical measurements of stellar abundances in early-type galaxies from the Sloan Digital Sky Survey 26 , where velocity dispersion dependence and systematic errors of 0.05 dex are taken into account in the error bars. Si is not shown because its abundance is highly sensitive to the velocity dispersion. S and Ar abundances are unavailable in the optical study. 


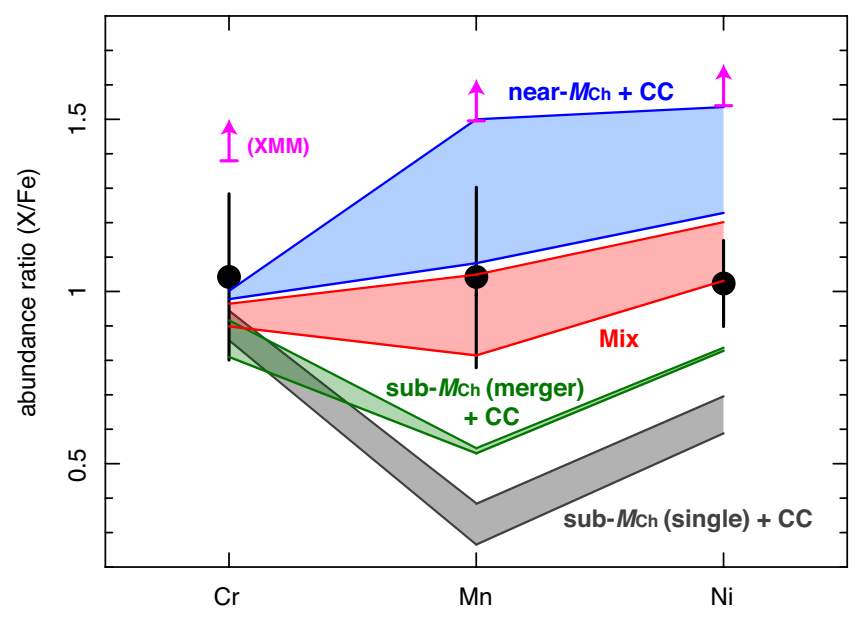

Figure 3: Comparison between the observed abundances and theoretical calculations for the Fe-peak elements. The black data points are identical to the red circles in Fig. 2, the SXS-measured abundance ratios relative to the solar abundances ${ }^{25}$. The error bars include both statistical uncertainty at a $1 \sigma$ confidence level and systematic uncertainty. The magenta arrows indicate the $1 \sigma$ lower limit of the XMM-Newton measurements for the 44 objects ${ }^{11}$. The blue, green, and gray regions represent the theoretical predictions for SNe Ia from the near- $M_{\mathrm{Ch}}$ delayed-detonation explosion ${ }^{12}$, sub- $M_{\mathrm{Ch}}$ violent merget ${ }^{13}$, and single sub- $M_{\mathrm{Ch}} \mathrm{WD}^{14}$, respectively. In each model, contributions from $\mathrm{CC} \mathrm{SNe}^{27}$ are also taken into account (see text). The red region assumes equal contributions of the near- $M_{\mathrm{Ch}} \mathrm{SNe}$ Ia and sub- $M_{\mathrm{Ch}}$ violent mergers, providing a reasonable fit to the data (although the exact ratio between the two SN Ia types is subject to some uncertainties in the model details). 


\section{Methods}

Observations and Data Reduction: The Hitomi observations of the Perseus Cluster core were performed using the SXS in the sequences summarized in Extended Data Table 1. The SXS field of view (FoV) of each sequence is indicated in Extended Data Figure 1. The data from the first four sequences, whose aim points were almost identical, were used in our previous work as well 17 . The spacecraft attitude was slightly different for the last sequence, so that the nucleus of NGC 1275 was observed using the central pixels of the SXS. The aperture window consisting of a $262-\mu \mathrm{m}$ Be filter and several contaminant materials ${ }^{31}$ was not opened before the loss of the mission. This filter significantly attenuated the SXS effective area especially in the soft X-ray band, limiting the SXS bandpass to above $\sim 1.8 \mathrm{keV}$.

The data reduction was made with public tools provided by NASA's HEASARC. We used cleaned event data of the latest release version with the standard screening for the post-pipeline processes $^{32}$. The spectral analysis was performed using only GRADE Hp (high-resolution primary) events that have the best energy resolution. The redistribution matrix file (RMF) was generated with the extra-large size option, which accounts for all components of the line spread function, including the main peak, low-energy exponential tail, escape peaks, and electron-loss

continuum 3133 . The full width at half maximum (FWHM) of the main-peak component was measured to be $4.9 \mathrm{eV}$ for the ${ }^{55} \mathrm{Fe}$ calibration source $\mathrm{e}^{34]}$.

Additional Gain Correction: Because of the short life of the mission, opportunities for onboard calibration were limited. This caused some uncertainty in the detector gain (pulse height-energy conversion factors), particularly at the energies far from the $\mathrm{Mn} \mathrm{K} \alpha$ calibration lines at $5.9 \mathrm{keV}$ (in a calibration pixel irradiated by a ${ }^{55} \mathrm{Fe}$ source). We thus applied the following gain calibration and correction using the Perseus data themselves.

First, we model the $\mathrm{Fe} \mathrm{He} \alpha$ complex with an ionization equilibrium plasma for each pixel in each sequence (combining the second through fourth sequences in Extended Data Table 1, since these were parts of a continuous observation with almost identical instrumental conditions), and scaled the spectrum with a linear function such that the $\mathrm{Fe} \mathrm{He} \alpha$ energies match the theoretical values at the redshift of NGC $1275(z=0.01756)^{\sqrt{36}}$. We then merged the data of all the pixels and measured the X-ray energies of detected lines. The differences between the measured and theoretical energies are plotted in Extended Data Figure 2. The discrepancy, while small, increases toward lower and higher energies with respect to the calibration source line (i.e., $5.9 \mathrm{keV}$ ). We empirically fit these plots with a parabolic function, and then updated the pulse invariant spectral channel of each detected event using the derived coefficients. Readers are cautioned that this 
empirical correction should not be used outside of the range of the fit; in particular, the actual gain error must be almost zero at the energies near $0 \mathrm{eV}$. The data from all sequences were then combined to increase the photon statistics. Hereafter, we use this merged, gain-corrected spectrum. We also appropriately took into account the off-axis effective area of the Soft X-ray Telescope $(\mathrm{SXT})$ 37, when generating the telescope response for the merged data.

Spectral Analysis: We analyzed the SXS spectrum in the $1.8-9.0 \mathrm{keV}$ band with an energy bin size of $1 \mathrm{eV}$. The spectral fitting was made using the $\mathrm{C}$ statistic ${ }^{38}$ without subtracting any background component, since its level is negligibly low $\left(\sim 7 \times 10^{-4}\right.$ counts $\mathrm{s}^{-1} \mathrm{keV}^{-1}$ for the entire field of view), with even its strongest emission lines well below the source flux in the 1.8-9.0 $\mathrm{keV}$ energy band. In fact, no significant change in the spectral parameters is found, if we fit the source spectrum by simultaneously modeling the instrumental background data extracted from the night-Earth observations. The cosmic X-ray background is also negligible at this cluster core region; well below $1 \%$ of the source emission over the entire energy band 39 .

We fit the spectrum of the Perseus Cluster with a single-temperature optically thin thermal plasma model (bvvapec model in the XSPEC package) based on the latest version of the atomic database, AtomDB v.3.0.8 $8^{40}$. The fitted parameters included the electron temperature $\left(k T_{e}\right)$, redshift $(z)$, turbulence velocity $\left(v_{t}\right)$, emission measure, and the elemental abundances of $\mathrm{Si}, \mathrm{S}$, Ar, Ca, Cr, Mn, Fe, and Ni relative to the solar values (Extended Data Table 2) ${ }^{25}$. We included a power-law component and redshifted lines of Fe I $\mathrm{K} \alpha$ fluorescence ( $6.4 \mathrm{keV}$ at the rest frame) to

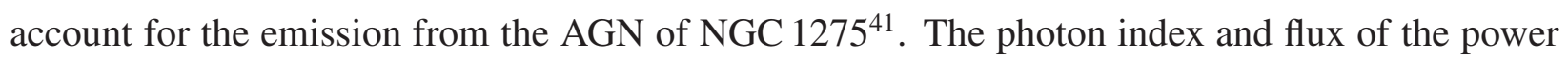
law component were determined to be $\Gamma \approx 1.9$ and $F_{2-10 \mathrm{keV}} \approx 3 \times 10^{-11} \mathrm{erg} \mathrm{s}^{-1} \mathrm{~cm}^{-2}$ using an AGN-dominated spectrum derived by SXS image analysis decomposing AGN and ICM emissions, and fixed to these values in the analysis of the ICM spectrum (Fig. 1) that was extracted from the entire SXS array. A foreground absorption column $\left(N_{\mathrm{H}}\right)$ was fixed at $1.38 \times 10^{21} \mathrm{~cm}^{-2} 42$. The possible effect of resonance scattering $(\mathrm{RS})^{\sqrt{1743}}$ was accounted by adding a Gaussian at the energy of the Fe XXV resonance line with a negative flux. Weak ${ }^{55} \mathrm{Fe}$ calibration source leakage events were taken into account by adding narrow Gaussians at the theoretical energies of the Mn K $\alpha$ lines, although this has no impact on our analysis results. With this model (hereafter "Model A1"), we obtained best-fit values of $k T_{e}=3.97 \pm 0.02 \mathrm{keV}$ and the absolute Fe abundance (i.e., the Fe/H number ratio relative to the solar values) of $0.63 \pm 0.01$ solar, with a C-statistic and $\chi^{2}$ of 7483 and 7862, respectively (7180 degrees of freedom). The relative abundances of the other elements (with respect to $\mathrm{Fe}$ ) are shown in Extended Data Figure 3. Note that the uncertainty in our gain correction is less than $1 \mathrm{eV}$ at energies near the $\mathrm{Mn} \mathrm{K} \alpha$ calibration lines (Extended Data Figure 2), and thus its effect is negligible for the determination of the Fe-peak element abundances. 
We carefully estimated systematic uncertainties in the measured abundances by introducing different models and assumptions. First, we excluded the RS correction, i.e., the negative-flux Fe XXV line (Model A2). This did not substantially change the relative abundances, confirming suggestions in previous work on this object 3941 . We also fit the spectrum with two-temperature models, with and without the RS effect (Models A3 and A4, respectively). In these models, the parameters other than the temperatures and emission measures were linked between the two components. We obtained best-fit temperatures of $k T_{e 1}=4.04 \pm 0.05 \mathrm{keV}$ and $k T_{e 2}=1.60$ $\pm 0.27 \mathrm{keV}$ with $2-10 \mathrm{-keV}$ flux ratio $\left(F_{1} / F_{2}\right)$ of 33.5 for Model A3, and similar values for Model A4. This indicates that the 4-keV component dominates over the entire SXS band and that the one-temperature modeling is already a good approximation for the observed region in this bandpass, although the presence of a multi-temperature plasma was previously inferred for this cluster $^{4344}$. already a good approximation for the observed region in this bandpass, although the presence of a multi-temperature plasma was previously inferred for this cluster ${ }^{4344}$. We also treated the absorption columns and the AGN spectral index and flux as free parameters, and confirmed no significant change in the relative abundances among the Fe-peak elements. Finally, we used the SPEX atomic code v.3.03 $3^{45}$ to fit the same spectral data with the same model components and assumptions (Models S1-S4, equivalent to Models A1-A4, respectively). The measured abundance ratios for each model are summarized in Extended Data Figure 3. The ranges between the minimum and maximum values among Models A1-A4 and S1-S4 are given in Fig. 2 as the uncertainty for the abundance of each element. The systematic uncertainties owing to the different atomic codes and assumptions are larger than the statistical errors but reasonably small for most of the elements. All the metal abundances are found to be fairly consistent with the solar values ${ }^{25}$. There are no significant differences in abundances derived from analysis of a region excluding the $2^{\prime} \times 2^{\prime}$ box centered on the AGN of NGC 1275 .

We have found that the abundance ratios of $\mathrm{Cr} / \mathrm{Fe}, \mathrm{Mn} / \mathrm{Fe}$, and $\mathrm{Ni} / \mathrm{Fe}$ are systematically lower than those determined in recent XMM-Newton studies ${ }^{11}$. Because an old plasma model (SPEX v.2.05) was used in this previous work, we also fit the SXS spectrum using that model for direct comparison. The results from one- and two-temperature modeling with the RS correction are given in Extended Data Figure 3 (Models $S^{\prime} 1$ and $S^{\prime} 3$, respectively) and Extended Data Figure 4 (red diamonds) with the combined uncertainty ranges. $\mathrm{Cr}$ and $\mathrm{Mn}$ abundances are not presented, because the SPEX v.2.05 atomic code does not contain emission from these elements - in the previous work, abundances of these elements were calculated by referring to emissivity data in an early development version of SPEX v.3. The Ni abundance determined from this old atomic model is slightly higher than from the latest one (SPEX v.3.03), but still lower than the XMM-Newton results. In fact, there is little difference in the Ni-He $\alpha$ emissivity itself between SPEX v.2.05 and 
v.3.03. We find significant differences between the two SPEX versions in the line emissivities of Fe XXIV and Fe XXV complex at the rest frame energies of 7.6-7.9 keV. Given that these emission cannot be separated from the Ni resonance line in CCD spectra, the Ni abundance might have been biased in the previous measurements.

Since $\mathrm{Cr}$ and $\mathrm{Mn}$ are rarely detected from individual objects with CCD observations, it is not obvious whether the supersolar abundances derived from the integrated XMM-Newton data of the 44 objects are real or biased. On the other hand, Suzaku observations (with similar CCDs) detected these elements from the same Perseus core region as in this work ${ }^{39}$. The Suzaku-measured abundances, converted to the same scale using the up-to-date solar abundance table ${ }^{25}$, are compared with the Hitomi and XMM-Newton results in Extended Data Figure 4 (green squares). This earlier measurement of the $\mathrm{Mn} / \mathrm{Fe}$ ratio is significantly lower than ours, further motivating the following demonstration of the robustness of our measurements compared to that of CCD observations.

Extended Data Figure 5(a) shows the SXS spectrum near the Cr and Mn emission lines, of which equivalent widths are only a few electron volts. The red line indicates our best-fit model (Model A1) but with $\mathrm{Cr}$ and Mn abundances set to zero. As shown in the bottom panel of the figure, the photon count ratios between the line peak and the local continuum level is $\sim 1.2$ for these weak emission lines in this high-resolution spectrum. Extended Data Figure 5(b) is a similar plot but the spectrum is convolved to the resolution of CCDs using a representative XMM-Newton response function. Unlike the SXS spectrum, the peak-to-continuum level ratios for the $\mathrm{Cr}$ and Mn emission are extremely low (only a few percent above unity). Moreover, the emission lines no longer have a sharp profile, implying the difficulty in separating lines from continuum. In fact, if we fit this simulated CCD spectrum with a model with $1 \%$ higher/lower continuum normalization, the line components with their broad profiles 'compensate' for the excess/lack of continuum flux by requiring $\sim 50 \%$ lower/higher values of the $\mathrm{Cr} / \mathrm{Fe}$ and $\mathrm{Mn} / \mathrm{Fe}$ abundance ratios. The high resolution SXS spectrum is much less subject to such systematic uncertainties, since the line and continuum intensities are measured almost independently and hence a slight over- or under-estimation of the continuum level has little effect on the abundance measurement. This point is more quantitatively illustrated in Extended Data Figure 6, the result of our test analysis.

Comparison with SN Nucleosynthesis Models: The measured abundances of the Fe-peak elements are compared with theoretical predictions to address the nature of SNe Ia that likely contributed to the chemical enrichment in the Perseus Cluster. As prototype SN Ia models, we select the latest three-dimensional calculations "N100" 12 and " $1.1 \_0.9$ " 13 . The former assumes a delayed-detonation explosion of a near- $M_{\mathrm{Ch}}$ WD with 100 deflagration ignition sites. The 
latter assumes the violent merger of two sub- $M_{\mathrm{Ch}}$ WDs with masses of $1.1 M_{\odot}$ and $0.9 M_{\odot}$ and subsequent full detonation of the primary (more massive) WD. Both models successfully replicate typical observables of SNe Ia, including the average maximum brightness and synthesized ${ }^{56} \mathrm{Ni}$ mass of $\sim 0.6 M_{\odot}$. The pre-explosion WD is composed of $47.5 \%{ }^{12} \mathrm{C}, 50 \%{ }^{16} \mathrm{O}$, and $2.5 \%{ }^{22} \mathrm{Ne}$ by mass, which corresponds to nearly solar metallicity for the progenitor. As another example of a sub- $M_{\mathrm{Ch}}$ explosion, we choose the " $10 \mathrm{HC}$ " mode1 ${ }^{14}$, which assumes an explosion of a single C-O WD with a mass of $1.0 M_{\odot}$ accreting helium at a rate $\dot{M}=4.0 \times 10^{-8} M_{\odot} \mathrm{yr}^{-1}$. An initial detonation ignited at the helium layer triggers a second detonation in the $\mathrm{CO}$ core, resulting in a complete explosion of the WD with a kinetic energy of $1.2 \times 10^{51}$ erg and ${ }^{56} \mathrm{Ni}$ mass of $\sim 0.64 M_{\odot}$, as typically inferred for SNe Ia.

To account for the CC SN contributions, we consider mass-dependent yields ${ }^{27}$ weighted by the Salpeter IMF $(\alpha=2.35)$, with the assumption that $50 \%$ of $\geq 25 M_{\odot}$ massive stars explode as hypernovae. Since SNe Ia efficiently produce $\mathrm{Fe}$, whereas $\mathrm{SNe} \mathrm{CC}$ dominate $\alpha$-element production, the SXS spectra we extracted might be used to constrain the $\mathrm{SN} \mathrm{Ia/CC}$ ratio in the Perseus Cluster. However, we instead allow a conservatively wide range for the CC SN fraction, $f_{\mathrm{CC}} \equiv N_{\mathrm{CC}} /\left(N_{\mathrm{Ia}}+N_{\mathrm{CC}}\right)=0.6-0.9^{29 \mid 19] 282946477}$, rather than determining the actual $f_{\mathrm{CC}}$ value. This choice was made because (1) the lighter elements that are most sensitive to $f_{\mathrm{CC}}$ (i.e., $\mathrm{O}, \mathrm{Ne}, \mathrm{Mg}$ ) were not detected due to the attenuation of soft $\mathrm{X}$-rays by the closed aperture window; (2) the measured abundances of the intermediate $\alpha$-burning elements, unlike those of the Fe-peak elements, are dominated by systematic, rather than the statistical, uncertainties (Extended Data Figure 3); and (3) the primary origins of $\mathrm{Ar}$ and $\mathrm{Ca}$ is currently under debate ${ }^{3 / 48}$. Future high-resolution X-ray spectroscopy with sensitivity to softer X-rays will improve the accuracy of the abundances of the lighter elements, as well as of the ICM spectral model, hence enabling better constrains on the $\mathrm{SN} \mathrm{Ia/CC}$ ratio. We emphasize that, in contrast to the intermediate $\alpha$-burning elements, the abundances of the Fe-peak elements are robustly determined with little model dependency (Extended Data Figure 3). As a result, the main conclusions of this paper are not affected by any of the issues described above.

The abundance ratios predicted by the model calculations are given in Fig. 3. Because of the efficient electron capture as well as the low entropy freeze-out from nuclear statistical equilibrium ${ }^{5}$, higher abundances of $\mathrm{Mn}$ and $\mathrm{Ni}$ are expected in the near- $M_{\mathrm{Ch}} \mathrm{SNe} \mathrm{Ia}$. We also test other combinations of SN models as well as different IMF slopes (for CC SNe). Extended Data Table 3 summarizes the mass ratios among the Fe-peak elements and Fe yields (in $M_{\odot}$ ) predicted by the various SN Ia models we investigated $46[12,14,49-53$. Since this paper exclusively discusses the products of electron capture, we consider only recent calculations that were based on up-to-date 
weak interaction rates 54 . For CC SN models, we use different IMF slopes $(\alpha=2.0$ and 2.7) and assume that all 10-50 $M_{\odot}$ stars explode as normal SNe without any hypernova contribution. These results are summarized in Extended Data Table 4. We reach essentially the same conclusion described in the main text, i.e., higher mass ratios of $\mathrm{Mn} / \mathrm{Fe}$ and $\mathrm{Ni} / \mathrm{Fe}$ are always expected from near- $M_{\mathrm{Ch}} \mathrm{SNe}$ Ia (Extended Data Table 3), and a combination of near- $M_{\mathrm{Ch}}$ and sub- $M_{\mathrm{Ch}} \mathrm{SNe}$ Ia naturally explains the observed abundance pattern of the Fe-peak elements independently of contributions from CC SNe (Extended Data Table 4).

Data and Code Availability: The observational data analysed during the current study are available in NASA's HEASARC repository (https://heasarc.gsfc.nasa.gov). The atomic codes utilized in this study are also available online (AtomDB: http://www.atomdb.org/, SPEX: https://www.sron.nl/astrophysics-spex). 


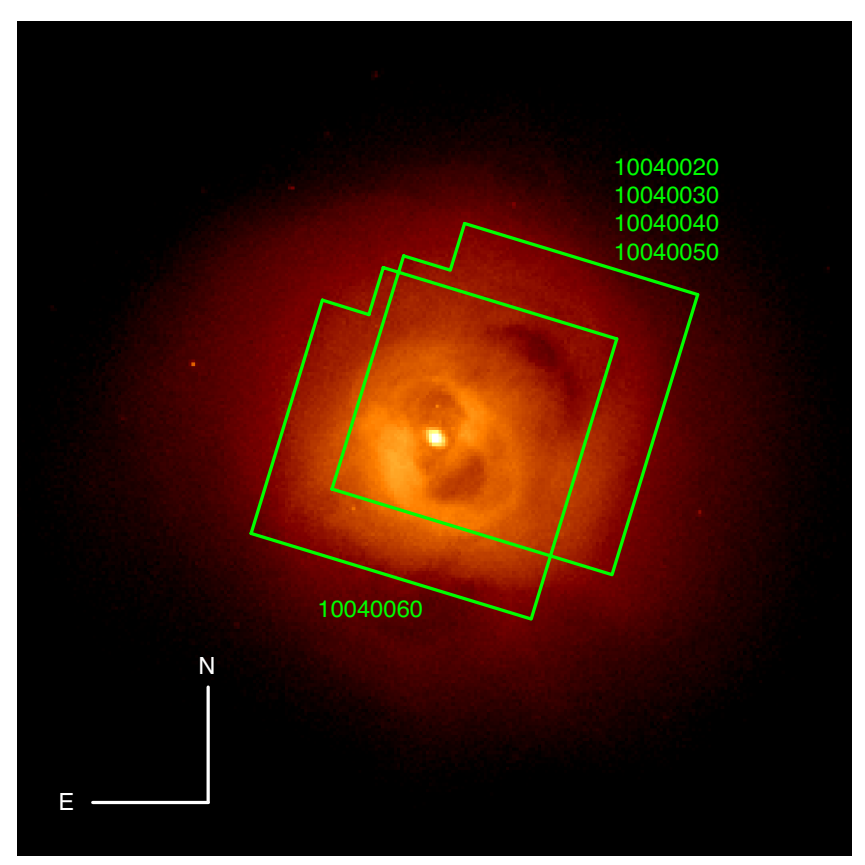

Extended Data Figure 1: The SXS FoV overlaid on a Chandra image. The corresponding Sequence IDs of the Hitomi observations are given. Each side of the SXS has an angular size of $3^{\prime}(\approx 64 \mathrm{kpc})$.
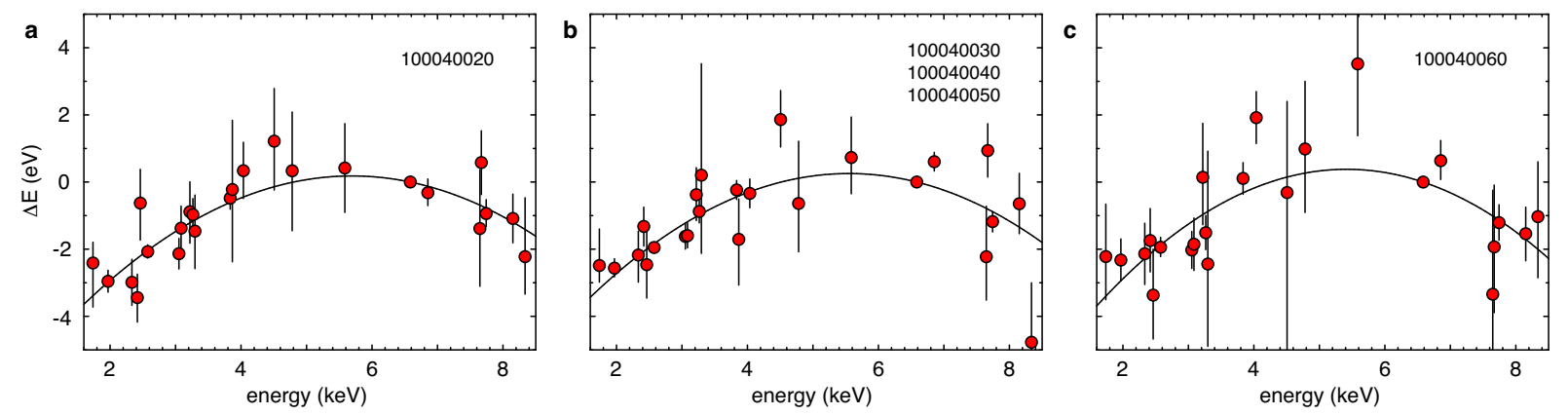

Extended Data Figure 2: Additional gain correction. The data points indicate the difference between the measured and theoretical energies $\left(\Delta E=E^{\prime}-E_{0}\right.$, where $E^{\prime}$ and $E_{0}$ are measured and theoretical energies, respectively) of each detected line at the given X-ray energy. The best-fit parabolic functions are given as the solid curves. The error bars correspond to the $1 \sigma$ confidence level. Panels (a), (b), and (c) are the results from Sequence 100040020, 100040030-50 (combined), and 100040060 , respectively. 

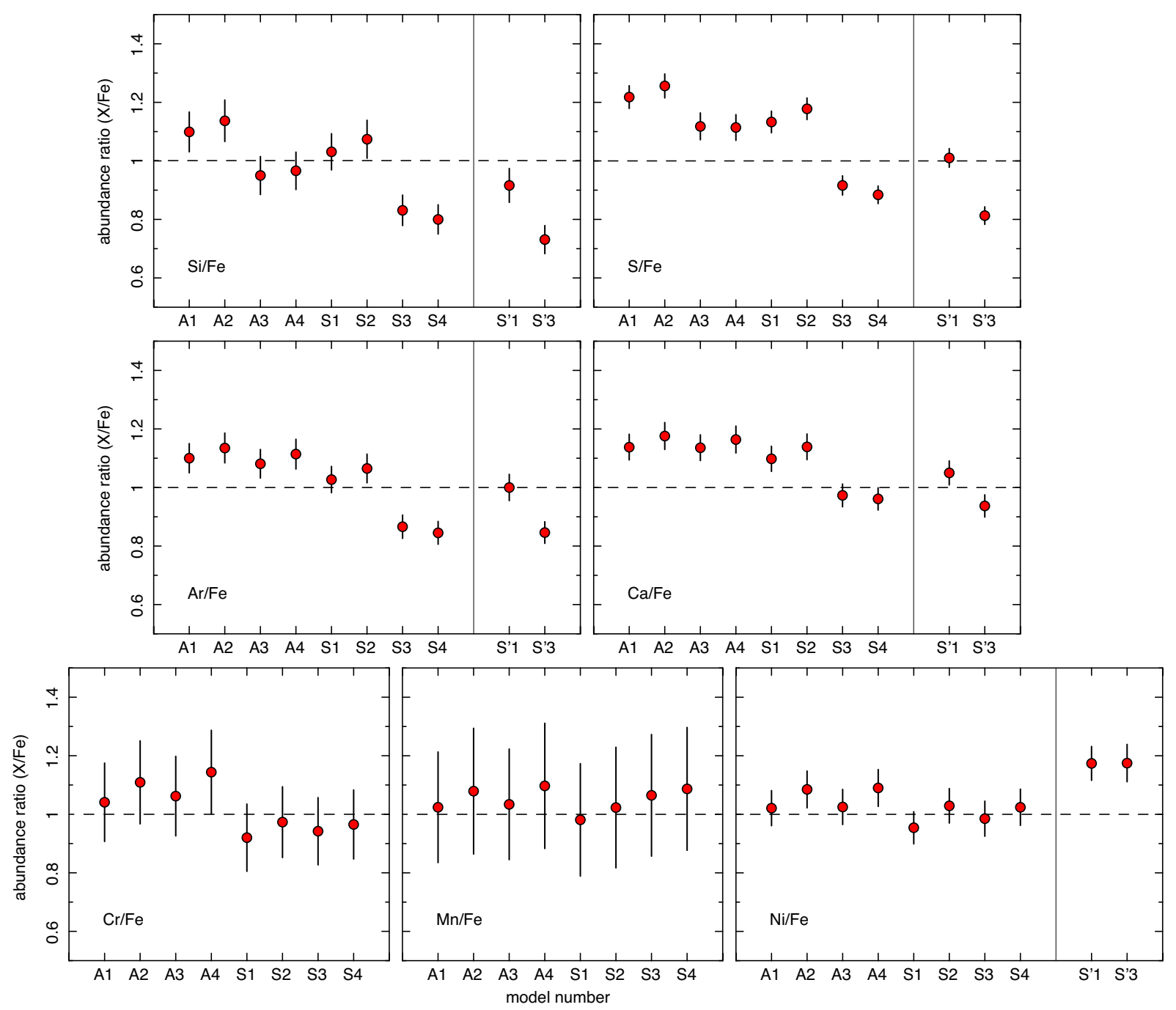

Extended Data Figure 3: Elemental abundances measured with different model assumptions. " $A$ " and "S" indicate the results for the atomic codes AtomDB v.3.0.8 and SPEX v.3.03, respectively; " $\mathrm{S}$ " an old atomic model (SPEX v.2.05 that does not contain $\mathrm{Cr}$ and Mn line data). Numerical designations are as follows. 1: one-temperature fit with the Fe XXV RS effect. 2: one-temperature fit without the RS effect. 3: two-temperature fit with the RS effect. 4: two-temperature fit without the RS effect. The error bars are at a $1 \sigma$ confidence level. 


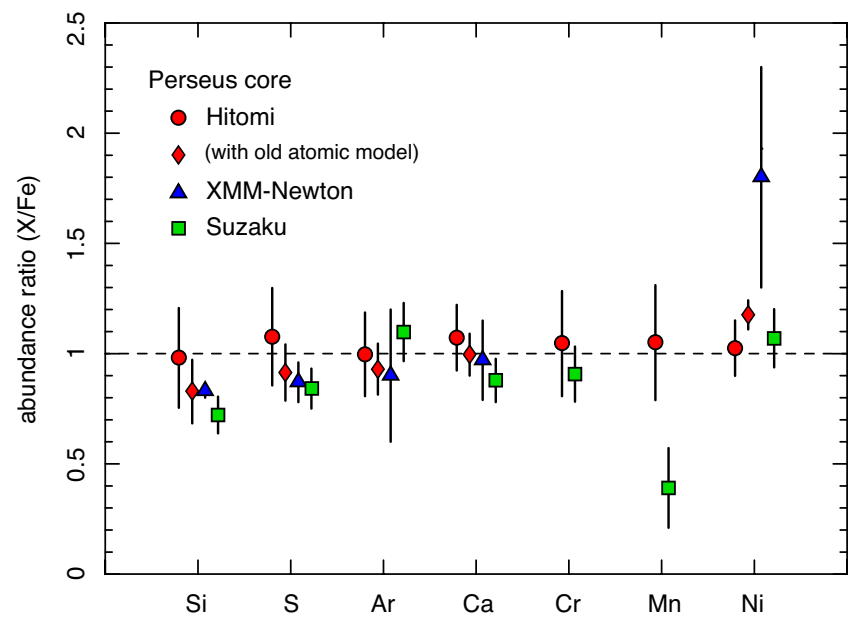

Extended Data Figure 4: Elemental abundances of the Perseus Cluster core compared among $\mathbf{X}$-ray measurements. The values are relative to the solar abundances ${ }^{25}$ with respect to Fe. The red circles are identical to those in Fig. 2 (in main body), representing the SXS measurements with error bars including both $1 \sigma$ statistical uncertainty and systematic uncertainty. The red diamonds are the SXS measurement with an outdated atomic model that was used in the previous XMM-Newton results. The blue triangles represent the XMM-Newton results $\frac{11}{}$, identical to those in Fig. 2. The green squares are abundances obtained by Suzaku observations of the innermost $2^{\prime}$ region of the Perseus Cluster ${ }^{\sqrt{39}}$ but are converted relative to the updated solar abundance table $\mathrm{e}^{25}$ for direct comparison with the other measurements. The error bars are also converted to the statistical uncertainty at a $1 \sigma$ confidence level. 

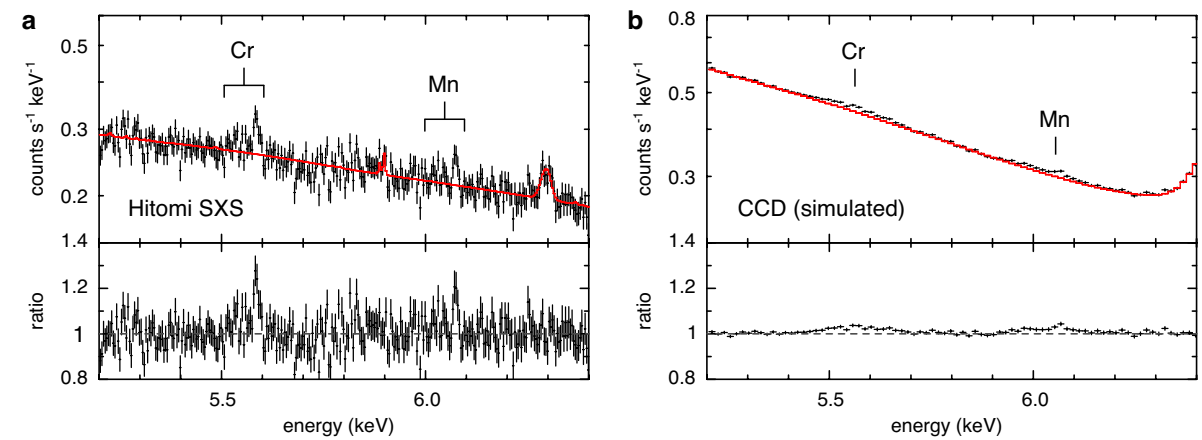

Extended Data Figure 5: Weak emission lines at different energy resolutions. (a) SXS spectrum of the Perseus Cluster around the $\mathrm{Cr}$ and Mn emission. The red line is the best-fit model (Model A1) but the $\mathrm{Cr}$ and $\mathrm{Mn}$ abundances are set to zero. The bottom panel shows the ratio between the data and model. The error bars correspond to the $1 \sigma$ confidence level. (b) Simulated spectrum at the energy resolution of the XMM-Newton MOS1 detector (representative of CCD data), where the best-fit model for the SXS data and sufficiently long exposure time (4 Ms) are assumed. This comparison demonstrates the robustness of our measurements of the weak emission lines with high resolution spectroscopy (see Methods for details). 

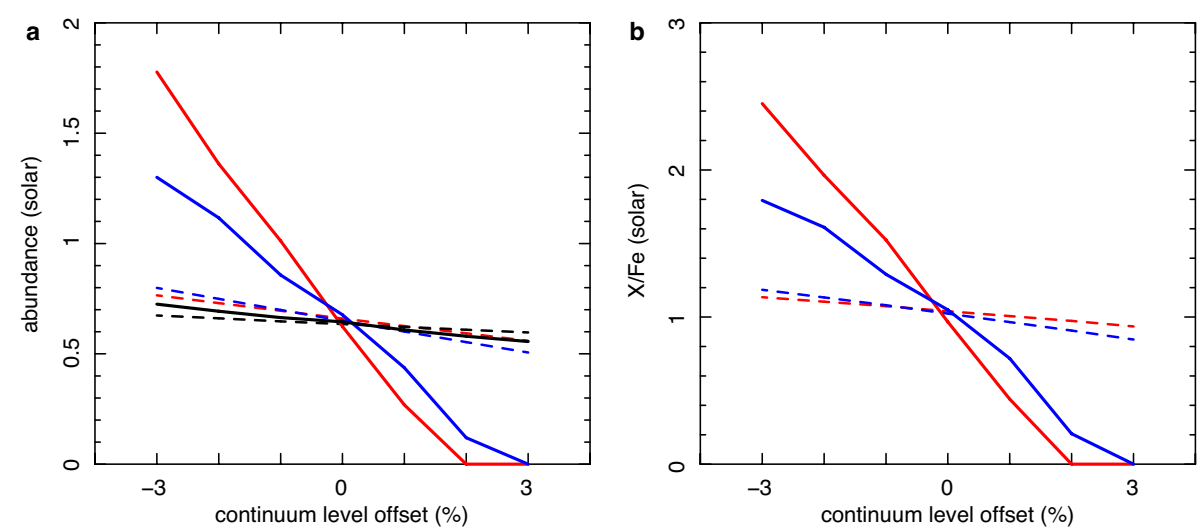

Extended Data Figure 6: Effect of potential bias in the continuum level estimate on the abundance measurement using weak emission lines. (a) Abundances of $\mathrm{Cr}$ (red), Mn (blue), and Fe (black) determined by intentionally giving a small offset to the continuum normalization (within $\pm 3 \%$ of the measured value). The solid and dashed lines are obtained from our test analysis of the simulated CCD spectrum (Extended Data Figure 5(b)) and the Hitomi spectrum, respectively. This illustrates that the CCD measurement of $\mathrm{Cr}$ and $\mathrm{Mn}$ abundances is sensitive to the accuracy of the continuum level determination because of the weakness of the emission and the low spectral resolution. The Fe abundance is less subject to such uncertainty even in the CCD measurement owing to the much larger equivalent width of the emission. (b) Abundance ratios of $\mathrm{Cr} / \mathrm{Fe}$ (red) and $\mathrm{Mn} / \mathrm{Fe}$ (blue) calculated using the values in panel (a) as a function of offset in the continuum level. 


\begin{tabular}{lcccc}
\hline Sequence ID & Observation Start Time & $\begin{array}{c}\text { Pointing R.A. } \\
\text { (deg) }\end{array}$ & $\begin{array}{c}\text { Pointing Dec. } \\
\text { (deg) }\end{array}$ & $\begin{array}{c}\text { Exposure } \\
\text { Time (ks) }\end{array}$ \\
\hline 100040020 & $2016-02-2502: 14: 12$ & 49.9316 & 41.5194 & 97.44 \\
100040030 & $2016-03-0402: 17: 32$ & 49.9324 & 41.5201 & 72.51 \\
100040040 & $2016-03-0512: 00: 15$ & 49.9321 & 41.5199 & 68.13 \\
100040050 & $2016-03-0619: 37: 59$ & 49.9323 & 41.5215 & 5.45 \\
100040060 & $2016-03-0622: 56: 19$ & 49.9510 & 41.5123 & 45.79 \\
\hline Total & & & & 289.32 \\
\hline
\end{tabular}

Extended Data Table 1: Summary of the observations. Sequences 100040030, 40, and 50 are continuous observations, and separated just for the data processing reason.

\begin{tabular}{lc}
\hline Element & Relative Number \\
\hline $\mathrm{H}$ & 1.00 \\
$\mathrm{He}$ & $9.71 \times 10^{-2}$ \\
$\mathrm{Si}$ & $3.85 \times 10^{-5}$ \\
$\mathrm{~S}$ & $1.62 \times 10^{-5}$ \\
$\mathrm{Ar}$ & $3.57 \times 10^{-6}$ \\
$\mathrm{Ca}$ & $2.33 \times 10^{-6}$ \\
$\mathrm{Cr}$ & $5.05 \times 10^{-6}$ \\
$\mathrm{Mn}$ & $3.56 \times 10^{-6}$ \\
$\mathrm{Fe}$ & $3.27 \times 10^{-5}$ \\
$\mathrm{Ni}$ & $1.89 \times 10^{-6}$ \\
\hline
\end{tabular}

Extended Data Table 2: Solar abundance table $\mathrm{e}^{\mathbf{2 5}}$ referred in this work. 


\begin{tabular}{|c|c|c|c|c|c|}
\hline Model & $\mathrm{Cr} / \mathrm{Fe}$ & $\mathrm{Mn} / \mathrm{Fe}$ & $\mathrm{Ni} / \mathrm{Fe}$ & $\begin{array}{c}\text { Fe mass } \\
\left(M_{\odot}\right)\end{array}$ & Reference \\
\hline \multicolumn{6}{|c|}{ Near- $M_{\mathrm{Ch}} \mathrm{SN}$ la models } \\
\hline N40 & 0.012 & 0.016 & 0.095 & 0.78 & 12 \\
\hline N100 & 0.014 & 0.018 & 0.10 & 0.74 & 12 \\
\hline N150 & 0.015 & 0.020 & 0.11 & 0.71 & 12 \\
\hline N40def & 0.0092 & 0.022 & 0.14 & 0.44 & 49 \\
\hline N100def & 0.0094 & 0.022 & 0.14 & 0.47 & 49 \\
\hline N150def & 0.0094 & 0.022 & 0.14 & 0.50 & 49 \\
\hline W7 & 0.0069 & 0.0088 & 0.16 & 0.76 & 4 \\
\hline CDEF & 0.0092 & 0.019 & 0.22 & 0.39 & 4 \\
\hline CDDT & 0.0098 & 0.017 & 0.21 & 0.36 & 4 \\
\hline ODDT & 0.016 & 0.011 & 0.12 & 0.65 & 4 \\
\hline c3_2d_512 & 0.0078 & 0.018 & 0.22 & 0.32 & 50 \\
\hline c3_2d_256 & 0.0084 & 0.015 & 0.21 & 0.41 & 50 \\
\hline c3_3d_256 & 0.0082 & 0.013 & 0.20 & 0.41 & 50 \\
\hline b5_3d_256 & 0.011 & 0.011 & 0.16 & 0.40 & 50 \\
\hline b30_3d_768 & 0.0060 & 0.012 & 0.20 & 0.53 & 50 \\
\hline DDTa & 0.019 & 0.020 & 0.099 & 0.72 & 51 \\
\hline \multicolumn{6}{|c|}{ Sub- $M_{\mathrm{Ch}}$ SN la models } \\
\hline $1.1 \_0.9$ & 0.011 & 0.0059 & 0.050 & 0.65 & 13 \\
\hline $10 \mathrm{HC}$ & 0.012 & 0.0023 & 0.032 & 0.63 & 14 \\
\hline 10HCD & 0.028 & 0.0034 & 0.037 & 0.61 & 14 \\
\hline $10 \mathrm{HD}$ & 0.018 & 0.0025 & 0.041 & 0.65 & 14 \\
\hline $11 \mathrm{HD}$ & 0.0076 & 0.0014 & 0.038 & 0.76 & 14 \\
\hline $10 \mathrm{~B}$ & 0.017 & 0.0022 & 0.039 & 0.73 & 14 \\
\hline $10 \mathrm{C}$ & 0.014 & 0.0021 & 0.044 & 0.69 & 14 \\
\hline $10 \mathrm{D}$ & 0.0098 & 0.0017 & 0.046 & 0.74 & 14 \\
\hline $9 \mathrm{~B}$ & 0.026 & 0.0034 & 0.050 & 0.61 & 14 \\
\hline $9 \mathrm{C}$ & 0.021 & 0.0028 & 0.040 & 0.64 & 14 \\
\hline 9D & 0.018 & 0.0024 & 0.044 & 0.66 & 14 \\
\hline $1.06 M_{\odot}$ & 0.031 & 0.0059 & 0.059 & 0.76 & $6(52)$ \\
\hline $0.97 M_{\odot}$ & 0.013 & 0.0092 & 0.051 & 0.58 & $6(52)$ \\
\hline $0.8 M_{\odot}+0.6 M_{\odot}$ & 0.017 & 0.0027 & 0.019 & 0.41 & 53 \\
\hline
\end{tabular}

Extended Data Table 3: Mass ratios among the Fe-peak elements in SN Ia models. 


\begin{tabular}{|c|c|c|c|c|c|c|c|c|c|}
\hline Near- $M_{C h}$ & Sub- $M_{\mathrm{Ch}}$ & $\mathrm{CC}$ & IMF & $f_{\mathrm{CC}}$ & $f_{M_{c h}}$ & $f_{\mathrm{Fe}, \mathrm{la}}$ & $\mathrm{Cr} / \mathrm{Fe}$ & $\mathrm{Mn} / \mathrm{Fe}$ & $\mathrm{Ni} / \mathrm{Fe}$ \\
\hline \multicolumn{10}{|c|}{ Near- $M_{\mathrm{Ch}}$ SNe la only } \\
\hline N100 & - & $\mathrm{SN}+\mathrm{HN}$ & 2.35 & 0.60 & 1.0 & 0.85 & 0.98 & 1.51 & 1.54 \\
\hline N100 & - & $\mathrm{SN}+\mathrm{HN}$ & 2.35 & 0.90 & 1.0 & 0.49 & 1.00 & 1.08 & 1.23 \\
\hline N100 & - & $\mathrm{SN}+\mathrm{HN}$ & 2.0 & 0.60 & 1.0 & 0.84 & 0.99 & 1.50 & 1.54 \\
\hline N100 & - & $\mathrm{SN}+\mathrm{HN}$ & 2.0 & 0.90 & 1.0 & 0.47 & 1.04 & 1.07 & 1.23 \\
\hline N100 & - & $\mathrm{SN}+\mathrm{HN}$ & 2.7 & 0.60 & 1.0 & 0.86 & 0.97 & 1.52 & 1.55 \\
\hline N100 & - & $\mathrm{SN}+\mathrm{HN}$ & 2.7 & 0.90 & 1.0 & 0.50 & 0.98 & 1.10 & 1.24 \\
\hline N100 & - & SN only & 2.35 & 0.60 & 1.0 & 0.87 & 1.01 & 1.54 & 1.54 \\
\hline N100 & - & SN only & 2.35 & 0.90 & 1.0 & 0.52 & 1.14 & 1.17 & 1.21 \\
\hline \multicolumn{10}{|c|}{ Sub- $M_{C h}$ SNe la only } \\
\hline- & 1.1_0.9 & $\mathrm{SN}+\mathrm{HN}$ & 2.35 & 0.60 & 0.0 & 0.83 & 0.81 & 0.55 & 0.83 \\
\hline- & $1.1 \_0.9$ & $\mathrm{SN}+\mathrm{HN}$ & 2.35 & 0.90 & 0.0 & 0.45 & 0.92 & 0.53 & 0.83 \\
\hline- & $1.1 \_0.9$ & $\mathrm{SN}+\mathrm{HN}$ & 2.0 & 0.60 & 0.0 & 0.82 & 0.82 & 0.55 & 0.84 \\
\hline- & $1.1 \_0.9$ & $\mathrm{SN}+\mathrm{HN}$ & 2.0 & 0.90 & 0.0 & 0.44 & 0.95 & 0.54 & 0.84 \\
\hline- & $1.1 \_0.9$ & $\mathrm{SN}+\mathrm{HN}$ & 2.7 & 0.60 & 0.0 & 0.84 & 0.80 & 0.54 & 0.83 \\
\hline- & 1.1_0.9 & $\mathrm{SN}+\mathrm{HN}$ & 2.7 & 0.90 & 0.0 & 0.47 & 0.89 & 0.53 & 0.82 \\
\hline- & $1.1 \_0.9$ & SN only & 2.35 & 0.60 & 0.0 & 0.85 & 0.85 & 0.56 & 0.82 \\
\hline- & 1.1_0.9 & SN only & 2.35 & 0.90 & 0.0 & 0.48 & 1.05 & 0.59 & 0.77 \\
\hline- & $10 \mathrm{HC}$ & $\mathrm{SN}+\mathrm{HN}$ & 2.35 & 0.60 & 0.0 & 0.83 & 0.86 & 0.27 & 0.59 \\
\hline- & $10 \mathrm{HC}$ & $\mathrm{SN}+\mathrm{HN}$ & 2.35 & 0.90 & 0.0 & 0.45 & 0.95 & 0.38 & 0.69 \\
\hline- & $10 \mathrm{HC}$ & $\mathrm{SN}+\mathrm{HN}$ & 2.0 & 0.60 & 0.0 & 0.82 & 0.88 & 0.27 & 0.59 \\
\hline- & $10 \mathrm{HC}$ & $\mathrm{SN}+\mathrm{HN}$ & 2.0 & 0.90 & 0.0 & 0.43 & 0.98 & 0.39 & 0.71 \\
\hline- & $10 \mathrm{HC}$ & $\mathrm{SN}+\mathrm{HN}$ & 2.7 & 0.60 & 0.0 & 0.84 & 0.85 & 0.26 & 0.58 \\
\hline- & $10 \mathrm{HC}$ & $\mathrm{SN}+\mathrm{HN}$ & 2.7 & 0.90 & 0.0 & 0.46 & 0.92 & 0.37 & 0.68 \\
\hline- & $10 \mathrm{HC}$ & SN only & 2.35 & 0.60 & 0.0 & 0.85 & 0.90 & 0.28 & 0.57 \\
\hline- & $10 \mathrm{HC}$ & SN only & 2.35 & 0.90 & 0.0 & 0.48 & 1.09 & 0.43 & 0.63 \\
\hline \multicolumn{10}{|c|}{ Both contributions of near- and sub- $M_{\mathrm{Ch}} \mathrm{SNe}$ la } \\
\hline N100 & $1.1 \_0.9$ & $\mathrm{SN}+\mathrm{HN}$ & 2.35 & 0.60 & 0.5 & 0.84 & 0.90 & 1.05 & 1.21 \\
\hline N100 & $1.1 \_0.9$ & $\mathrm{SN}+\mathrm{HN}$ & 2.35 & 0.90 & 0.5 & 0.47 & 0.96 & 0.81 & 1.04 \\
\hline N100 & $1.1 \_0.9$ & SN only & 2.35 & 0.60 & 0.5 & 0.86 & 0.94 & 1.08 & 1.20 \\
\hline N100 & $1.1 \_0.9$ & SN only & 2.35 & 0.90 & 0.5 & 0.50 & 1.10 & 0.89 & 1.00 \\
\hline N100 & $10 \mathrm{HC}$ & $\mathrm{SN}+\mathrm{HN}$ & 2.35 & 0.60 & 0.5 & 0.84 & 0.92 & 0.93 & 1.10 \\
\hline N100 & $10 \mathrm{HC}$ & $\mathrm{SN}+\mathrm{HN}$ & 2.35 & 0.90 & 0.5 & 0.47 & 0.98 & 0.74 & 0.97 \\
\hline N100 & $10 \mathrm{HC}$ & SN only & 2.35 & 0.60 & 0.5 & 0.86 & 0.96 & 0.95 & 1.09 \\
\hline N100 & $10 \mathrm{HC}$ & SN only & 2.35 & 0.90 & 0.5 & 0.50 & 1.11 & 0.81 & 0.93 \\
\hline
\end{tabular}

Extended Data Table 4: Example calculations of SN nucleosynthesis models for comparison with the observation. The first three columns indicate the name and/or combination of SN models, and the fourth column the assumed index of the IMF. $f_{\mathrm{CC}}, f_{M_{\mathrm{ch}}}$, are $f_{\mathrm{Fe}, \mathrm{Ia}}$ the number fraction of CC SNe: $N_{\mathrm{CC}} /\left(N_{\mathrm{Ia}}+N_{\mathrm{CC}}\right)$, the number fraction of near- $M_{\mathrm{Ch}} \mathrm{SNe}$ Ia among the total number of SNe Ia: $N_{M_{\mathrm{ch}}} / N_{\text {Ia }}$, and the mass fraction of Fe originating from SNe Ia, respectively. The remaining columns indicate abundance ratios relative to the solar values 25 . 


\section{Supplementary Information}

Acknowledgements H. Yamaguchi thanks Drs. Ivo Seitenzahl, Rüdiger Pakmor, Eduardo Bravo, and Nozomu Tominaga for providing the SN nucleosynthesis models used in this paper. We thank the support from the JSPS Core-to-Core Program. We acknowledge all the JAXA members who have contributed to the ASTRO-H (Hitomi) project. All U.S. members gratefully acknowledge support through the NASA Science Mission Directorate. Stanford and SLAC members acknowledge support via DoE contract to SLAC National Accelerator Laboratory DE-AC3-76SF00515. Part of this work was performed under the auspices of the U.S. DoE by LLNL under Contract DE-AC52-07NA27344. Support from the European Space Agency is gratefully acknowledged. French members acknowledge support from CNES, the Centre National d'Etudes Spatiales. SRON is supported by NWO, the Netherlands Organization for Scientific Research. Swiss team acknowledges support of the Swiss Secretariat for Education, Research and Innovation (SERI). The Canadian Space Agency is acknowledged for the support of Canadian members. We acknowledge support from JSPS/MEXT KAKENHI grant numbers 15H00773, 15H00785, 15H02090, 15H03639, 15H05438, 15K05107, 15K17610, 15K17657, 16H00949, 16H06342, 16K05295, 16K05296, 16K05300, 16K13787, 16K17672, 16K17673, 21659292, 23340055, 23340071, 23540280, 24105007, 24540232, 25105516, 25109004, 25247028, 25287042, 25400236, 25800119, 26109506, 26220703, 26400228, 26610047, 26800102, JP15H02070, JP15H03641, JP15H03642, JP15H03642, JP15H06896, JP16H03983, JP16K05296, JP16K05309, and JP16K17667. The following NASA grants are acknowledged: NNX15AC76G, NNX15AE16G, NNX15AK71G, NNX15AU54G, NNX15AW94G, and NNG15PP48P to Eureka Scientific. H. Akamatsu acknowledges support of NWO via Veni grant. C. Done acknowledges STFC funding under grant ST/L00075X/1. A. Fabian and C. Pinto acknowledge ERC Advanced Grant 340442. P. Gandhi acknowledges JAXA International Top Young Fellowship and UK Science and Technology Funding Council (STFC) grant ST/J003697/2. Y. Ichinohe and K. Nobukawa are supported by the Research Fellow of JSPS for Young Scientists. N. Kawai is supported by the Grant-in-Aid for Scientific Research on Innovative Areas "New Developments in Astrophysics Through Multi-Messenger Observations of Gravitational Wave Sources". S. Kitamoto is partially supported by the MEXT Supported Program for the Strategic Research Foundation at Private Universities, 2014-2018. B. McNamara and S. Safi-Harb acknowledge support from NSERC. T. Dotani, T. Takahashi, T. Tamagawa, M. Tsujimoto and Y. Uchiyama acknowledge support from the Grant-in-Aid for Scientific Research on Innovative Areas "Nuclear Matter in Neutron Stars Investigated by Experiments and Astronomical Observations". N. Werner is supported by the Lendület LP2016-11 grant from the Hungarian Academy of Sciences. D. Wilkins is support by NASA through Einstein Fellowship grant number PF6-170160, awarded by the Chandra X-ray Center, operated by the Smithsonian Astrophysical Observatory for NASA under contract NAS8-03060 We thank contributions by many companies, including in particular, NEC, Mitsubishi Heavy Industries, Sumitomo Heavy Industries, and Japan Aviation Electronics Industry.

Finally, we acknowledge strong support from the following engineers. JAXA/ISAS: Chris Baluta, 
Nobutaka Bando, Atsushi Harayama, Kazuyuki Hirose, Kosei Ishimura, Naoko Iwata, Taro Kawano, Shigeo Kawasaki, Kenji Minesugi, Chikara Natsukari, Hiroyuki Ogawa, Mina Ogawa, Masayuki Ohta, Tsuyoshi Okazaki, Shin-ichiro Sakai, Yasuko Shibano, Maki Shida, Takanobu Shimada, Atsushi Wada, Takahiro Yamada; JAXA/TKSC: Atsushi Okamoto, Yoichi Sato, Keisuke Shinozaki, Hiroyuki Sugita; Chubu U: Yoshiharu Namba; Ehime U: Keiji Ogi; Kochi U of Technology: Tatsuro Kosaka; Miyazaki U: Yusuke Nishioka; Nagoya U: Housei Nagano; NASA/GSFC: Thomas Bialas, Kevin Boyce, Edgar Canavan, Michael DiPirro, Mark Kimball, Candace Masters, Daniel Mcguinness, Joseph Miko, Theodore Muench, James Pontius, Peter Shirron, Cynthia Simmons, Gary Sneiderman, Tomomi Watanabe; ADNET Systems: Michael Witthoeft, Kristin Rutkowski, Robert S. Hill, Joseph Eggen; Wyle Information Systems: Andrew Sargent, Michael Dutka; Noqsi Aerospace Ltd: John Doty; Stanford U/KIPAC: Makoto Asai, Kirk Gilmore; ESA (Netherlands): Chris Jewell; SRON: Daniel Haas, Martin Frericks, Philippe Laubert, Paul Lowes; U of Geneva: Philipp Azzarello; CSA: Alex Koujelev, Franco Moroso. 